\title{
DETRITAL ZIRCON EVIDENGE FROM BURMA FOR REORGANIZATION OF THE EASTERN HIMALAYAN RIVER SYSTEM
}

\author{
YU-HSUAN LIANG*, SUN-LIN CHUNG*,†, DUNYI LIU**, YIGANG XU***, \\ FU-YUAN WU ${ }^{\S}$, JIN-HUI YANG ${ }^{\S}$, YANBIN WANG**, and CHING-HUA LO*
}

\begin{abstract}
The first in situ $\mathrm{Hf}$ and $\mathrm{U}-\mathrm{Pb}$ isotope analyses of detrital zircons from Upper Miocene sandstone in the Inner-Burma Tertiary Basin, together with data of igneous zircons from the eastern Transhimalayan batholith from southern Tibet to Burma, enable us to study the sedimentary source to sink relation and river evolution around the eastern Himalayas. Among 47 out of 62 dated zircons from the Miocene sandstone that exhibit Cretaceous and Paleogene ${ }^{206} \mathrm{~Pb} /{ }^{238} \mathrm{U}$ ages, 24 grains have positive $\varepsilon_{\mathrm{Hf}}(\mathrm{T})$ isotope values up to +16 . Whilst zircons of such ages are common in the Transhimalayan plutons, those showing high $\varepsilon_{\mathrm{Hf}}(T)$ values have been observed so far only in the Gangdese batholith. Our results, therefore, support the notion that by Late Miocene time the Yarlu Tsangpo, which flows past the Gangdese batholith in southeastern Tibet, drained into the Irrawaddy River. We attribute this river routing to not only regional topographic control but also the dextral movement of the JialiGaoligong-Sagaing fault system that appears most active during the Middle Miocene. Subsequent reorganization of these mountain rivers was affiliated with headward erosion of the Brahmaputra River that eventually cut across the Namche Barwa Syntaxis and captured the Yarlu Tsangpo drainage to form the modern eastern Himalayan river system.
\end{abstract}

\section{INTRODUCTION}

Mountains formed by tectonic forces due to the India-Asia collision can influence regional drainage patterns, which in turn may have controlled the discharge of eroded sediments to the ocean (Brookfield, 1998; Zhang and others, 2001). Although how tectonic uplift, drainage system evolution, river erosion and alluvial deposition, interacted are debated (compare Molnar, 2003), they are widely considered as fundamental processes that have been shaping the landscape of the HimalayanTibetan orogen and surrounding areas in Cenozoic time (for example, Koons, 1995; Hallet and Molnar, 2001; Zeitler and others, 2001; Finlayson and others, 2002; Clark and others, 2004; Clift and others, 2004; Clift, 2006). For example, Clark and others (2004) analyzed the drainage pattern in East Asia and proposed that the Red River was an ancestral river that suffered progressive loss of drainage to the neighboring river systems due to Tibetan uplift and associated change in regional topography. Under this framework, the Yarlu Tsangpo (in Tibetan, "tsangpo" = river) in southeastern Tibet was once the upper reach of the Red River, before being captured by the Irrawaddy and then Brahmaputra Rivers (fig. 1). The timing of these river capture events, however, cannot be reconstructed from the topographic evidence alone.

In this paper, we report the first in situ U-Pb age dating and $\mathrm{Hf}$ isotope analyses of detrital zircons from a sandstone from the Inner-Burma Tertiary Basin and magmatic zircons from the Bomi-Chayu and Dianxi-Burma batholiths, part of the Transhimalayan plutons exposed around the eastern Himalayan syntaxis (fig. 1), by using the sensitive high-resolution ion microprobe (SHRIMP) and laser ablation-multicollectorinductively coupled plasma mass spectrometry (LA-MC-ICPMS), respectively. We combine these results with reported zircon $\mathrm{U}-\mathrm{Pb}$ and $\mathrm{Hf}$ isotope data from the

\footnotetext{
*Department of Geosciences, National Taiwan University, P.O. Box 13-318, Taipei 106, Taiwan, ROC

**Institute of Geology, Chinese Academy of Geological Sciences, Beijing, China

***Guangzhou Institute of Geochemistry, Chinese Academy of Sciences, Guangzhou, China

\$nstitute of Geology and Geophysics, Chinese Academy of Sciences, Beijing, China

tCorresponding author: E-mail: sunlin@ntu.edu.tw
} 


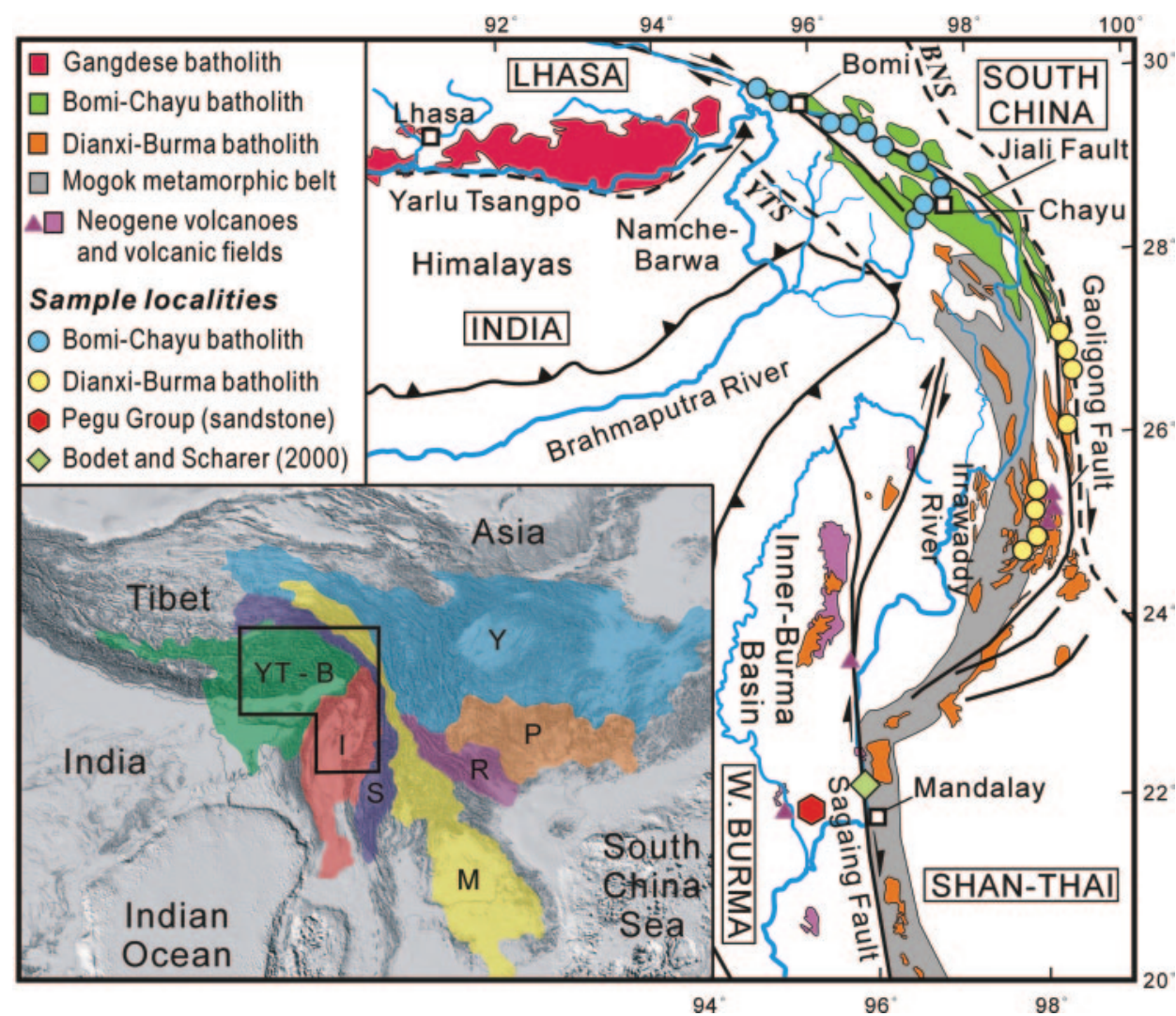

Fig. 1. Simplified geologic map showing the distribution of principal batholiths, faults and river systems around eastern Himalayas, together with sample localities of this study. BNS: Bangong Nujiang suture; YTS Yarlu Tsangpo suture. Inset denotes major drainage basins in East Asia, including: YT-B: Yarlu TsangpoBrahmaputra River; I: Irrawaddy River; S: Salween River; M: Mekong River; R: Red River; P: Pearl River; Y: Yangtze River.

Gangdese batholith, the largest plutonic complex in southeastern Tibet (Chu, ms, 2006; Chu and others, 2006), and those from riverbank sediments, the Irrawaddy River (Bodet and Schärer, 2000) to put forth the best available interpretation regarding the sedimentary source to sink relation. Although further detailed sampling in the future could produce other results, the present data set would still stand as an important constraint that allows us to not only test the existing hypotheses but also better understand the evolution of the eastern Himalayan river system.

\section{BACKGROUND OF THE STUDY}

Combined analyses of $\mathrm{U}-\mathrm{Pb}$ and $\mathrm{Hf}$ isotopes in single grains of detrital zircon from major rivers in the Southeast Asian continent was first performed by Bodet and Schärer (2000) to study regional crustal evolution and orogenic cycles. Among the important features reported, the Irrawaddy drainage was noted by the authors to be marked by the presence of young detrital zircons $(<90 \mathrm{Ma})$ that possess high $\varepsilon_{\mathrm{Hf}}(\mathrm{T})$ isotope values (up to +13.3 ) suggestive of juvenile mantle input, in contrast to the negative and apparently lower $\varepsilon_{\mathrm{Hf}}(\mathrm{T})$ values observed commonly in zircons from other Southeast Asian rivers. This Irrawaddy "anomaly" was believed to reflect a "large proportion of mantle derived magmas" resulting from young and major orogenic events such as 
the India-Asia collision (Bodet and Schärer, 2000). This conclusion was reached despite the fact that any attempt to carry out a sedimentary source to deposition analysis was merely impossible, because there was little knowledge about where young mantle derived magmas occur.

In this region, $\mathrm{U}-\mathrm{Pb}$ age data for single grains of zircon separates from riverbank sediments have been reported subsequently by two papers. These are (1) Campbell and others (2005) from the Ganges and Indus Rivers, and (2) Clift and others (2006a) from the Red and Mekong Rivers. Both studies, aiming at determining the source provenance and exhumation/erosion history, utilized additional dating techniques, that is, (U-Th)/He and fission track, which, relative to the U-Pb method, may provide low-temperature age information of the zircon grains dated. Besides, in the Himalayan sedimentary basins, investigations of the detrital records have been performed using various approaches and techniques (Najman, 2006; for a comprehensive review). All the above works, however, did not include Hf isotope analysis of detrital zircons.

The voluminous Transhimalayan plutons, now exposed largely in the Lhasa terrane of southern Tibet, have been generally regarded as resulting from northward subduction of the Neotethyan oceanic lithosphere along the South Asian continental margin before the closing of Tethys and/or onset of the India-Asia collision (Searle and others, 1987). Recently, as part of our ongoing study of the Transhimalayan magmatism, Chu (ms, 2006) and Chu and others (2006) reported SHRIMP U-Pb and LA-MC-ICPMS Hf isotope data of magmatic zircons from the Gangdese batholith that represents one of the largest Transhimalayan plutons in southeastern Tibet. The Gangdese zircons, aged between ca. 200 and $40 \mathrm{Ma}$, are overwhelmed by high $\varepsilon_{\mathrm{Hf}}(\mathrm{T})$ values from +18 to -4 (with $\sim 95 \%$ of them showing positive values; Chu, ms, 2006). These data shed light on the source of the specific zircons observed from the Irrawaddy riverbank sediments, which, if verified, would lend strong support to the topographic argument for a preexisting link between the Yarlu Tsangpo and Irrawaddy drainages proposed by Clark and others (2004). To comprehend the spatial and, in particular, temporal evolution of the river system, we carried out in this study new $\mathrm{U}-\mathrm{Pb}$ and $\mathrm{Hf}$ isotope analyses of magmatic zircons from the Bomi-Chayu and Dianxi-Burma batholiths, two important but previously poorly studied granitoid exposures around the eastern Himalayan syntaxis, in addition to the detrital zircon investigation in the Inner-Burma Basin.

SAMPLES AND METHODS

Zircons were separated from $\sim 3 \mathrm{~kg}$ samples by heavy-liquid and magnetic methods. The samples include granitic rocks from the Bomi-Chayu and Dianxi-Burma batholiths, and a sandstone from the uppermost Pegu Group located $\sim 60 \mathrm{~km}$ west of Mandalay city (fig. 1). In the Inner-Burma Tertiary Basin the change in sedimentation from the marine Pegu Group to the terrestrial Irrawaddy Group took place around the Miocene-Pliocene boundary (Bender, 1983). Hence, the depositional age of the studied sandstone sample BUR04-09 is regarded to be Late Miocene, say, ca. 5 to 10 Ma. All zircon U-Pb dating measurements were performed at the Beijing SHRIMP Center, Chinese Academy of Geological Sciences, following the analytical procedures reported in Chu and others (2006). In situ Hf isotope measurements were performed, later, on the dated spots of the zircons using the LAM-MC-ICPMS system at the Institute of Geology and Geophysics, Chinese Academy of Sciences. The diameter of the laser-ablated craters is $\sim 60 \mu \mathrm{m}$, about twice that of the spots made by SHRIMP dating. The analytical procedures were generally the same as those reported in Wu and others (2006), with a $193 \mathrm{~nm}$ excimer laser being attached to a Finnigan Neptune MC-ICPMS. 
The analytical results are summarized in tables 1,2 and 3 , in which, $\varepsilon_{\mathrm{Hf}}(\mathrm{T})$ values, the parts in $10^{4}$ deviation of initial Hf isotope ratios between the zircon sample and the chondritic reservoir, were calculated using the ${ }^{176} \mathrm{Lu}^{-176} \mathrm{Hf}$ decay constant reported in Söderlund and others (2004). In figures 2 and 3, the U-Pb age results of the Pegu sandstone and the Bomi-Chayu batholith, respectively, are shown using concordia diagrams. U-Pb ages of the Dianxi-Burma batholith, however, are from published data by Yang and others (2006). In figures 4 and 5, all zircon results are plotted in terms of $\varepsilon_{\mathrm{Hf}}(\mathrm{T})$ values versus $\mathrm{U}-\mathrm{Pb}$ ages.

\section{The Inner-Burma Sandstone}

62 grains of zircon separates from sample BUR04-09 were dated, with a random selection of zircon grains from all sizes and morphologies except for avoidance of those showing fractures or inclusions. While a large range of $\mathrm{U}-\mathrm{Pb}$ ages between 24 and $3151 \mathrm{Ma}$ was obtained (table 1; fig. 2), 48 dated grains $(\sim 78 \%)$ are $<200 \mathrm{Ma}$, including 16 and 31 grains that yielded Cretaceous and Paleogene ages, respectively. The $\mathrm{U}$ and Th concentrations vary strikingly $(\sim 5000-16 \mathrm{ppm})$, but most zircons have $\mathrm{Th} / \mathrm{U}$ ratios $>0.1$ (fig. 6) indicative of a magmatic origin (Hoskin and Schaltegger, $2003)$. Their overall $\varepsilon_{\mathrm{Hf}}(\mathrm{T})$ values also vary remarkably, that is, from +16 to -21 (table 1 ), but do not display correlations with the Th and U concentrations or ratios. Such a large isotopic variation is most clearly manifested by the Cretaceous and Paleogene zircons (fig. 5), among which 24 grains show positive $\varepsilon_{\mathrm{Hf}}(\mathrm{T})$ values and the remaining 23 grains have negative values.

\section{The Transhimalayan Batholiths}

100 grains of igneous zircons from 10 granitoids within the Bomi-Chayu batholith (fg. 1) were analyzed (table 2, figs. 3 and 4). Zircons from this batholith are mostly euhedral and long to short prismatic, with lengths of $\sim 150$ to $200 \mu \mathrm{m}$ and length-towidth ratios up to 3:1. Most zircon crystals are transparent, colorless to slightly brown and show oscillatory zoning typical of magmatic growth (Hoskin and Schaltegger, 2003). Zircons with rounded or ovoid shapes and complex internal textures are rare. Given the fact that ages of the dated zircons are young, weighted means of pooled ${ }^{206} \mathrm{~Pb} /{ }^{238} \mathrm{U}$ ages are taken to represent the crystallization ages of their host rocks, and these ages are presented in the concordia diagrams with uncertainties at two standard deviation $(2 \sigma)$ or 95 percent confidence level (fig. 3). The U-Pb ages, together with Hf isotope results, allow us to delineate two zircon groups, which are (1) those with ${ }^{206} \mathrm{~Pb} /{ }^{238} \mathrm{U}$ ages from ca. 106 to $137 \mathrm{Ma}$ and $\varepsilon_{\mathrm{Hf}}(\mathrm{T})$ values from +2.5 to -15.1 , and (2) those with ${ }^{206} \mathrm{~Pb} /{ }^{238} \mathrm{U}$ ages from ca. 50 to $80 \mathrm{Ma}$ and $\varepsilon_{\mathrm{Hf}}(\mathrm{T})$ values from -15.0 to -7.5 (fig. 4). Besides, there are two outliers showing the lowest $\varepsilon_{\mathrm{Hf}}(\mathrm{T})$ value (-27.3) and oldest age (162.1 Ma), respectively (table 2 and fig. 4 ).

Of the Dianxi-Burma batholith, 110 igneous zircon grains from 8 granitoids that have been U-Pb dated by Yang and others (2006) were subjected to in situ Hf isotope analysis (table 3). The results, plotted also in figure 4, identify similar groupings as those delineated by the Bomi-Chayu samples. More significantly, when comparing with the Gangdese data (fig. 4), the Hf isotope ratios of magmatic zircons from both these batholiths are much lower than those of the Gangdese batholith (Chu, ms, 2006; Chu and others, 2006) or Gangdese-derived zircons in the Yarlu Tsangpo riverbank sediments (Liang and others, 2004).

\section{DISCUSSION AND INTERPRETATIONS}

\section{The Gangdese "Fingerprint" in Zircon}

Our zircon U-Pb age results from the Bomi-Chayu and Dianxi-Burma batholiths support the general consensus that regards these batholiths as the easternmost 


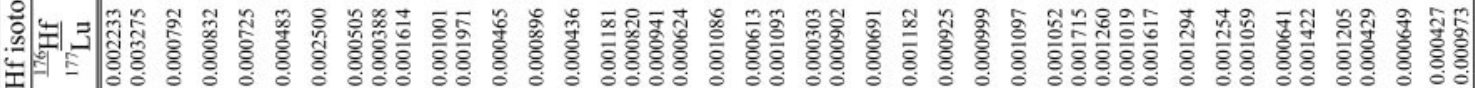

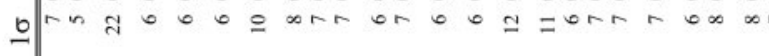

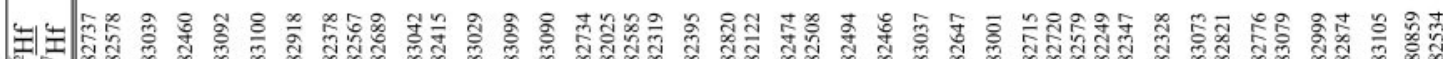

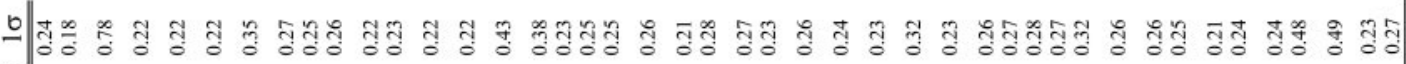

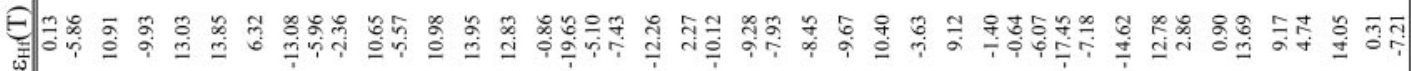

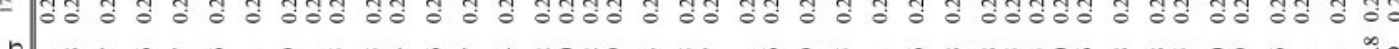

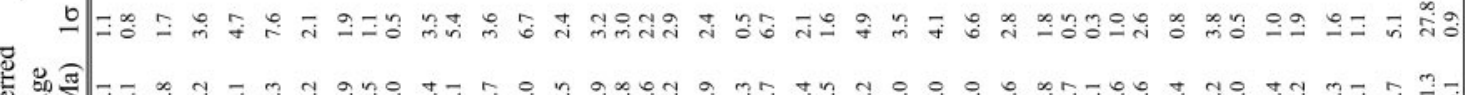
0 낭

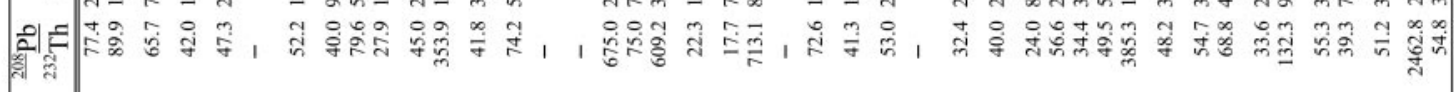

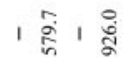

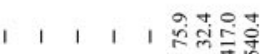

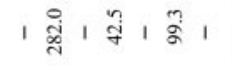
武放

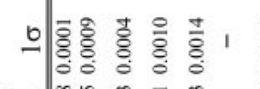

总

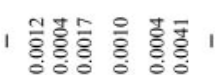

응 :

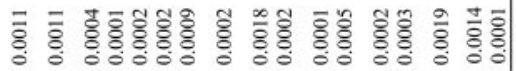

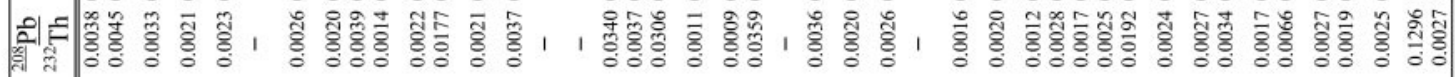

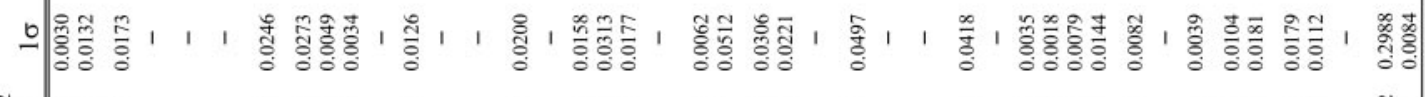

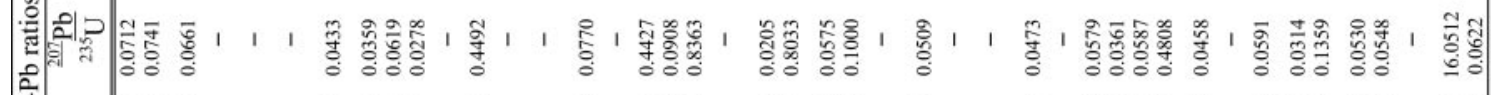
|

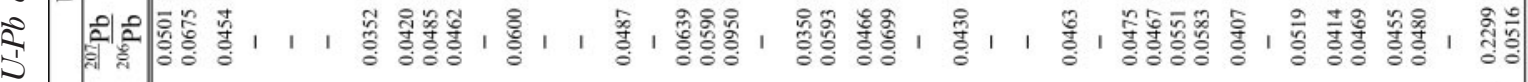

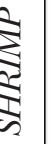

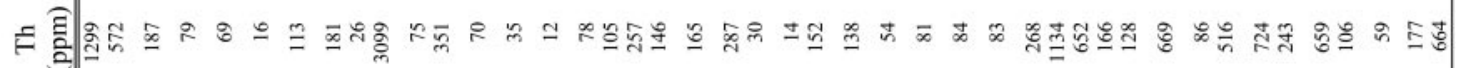

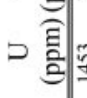




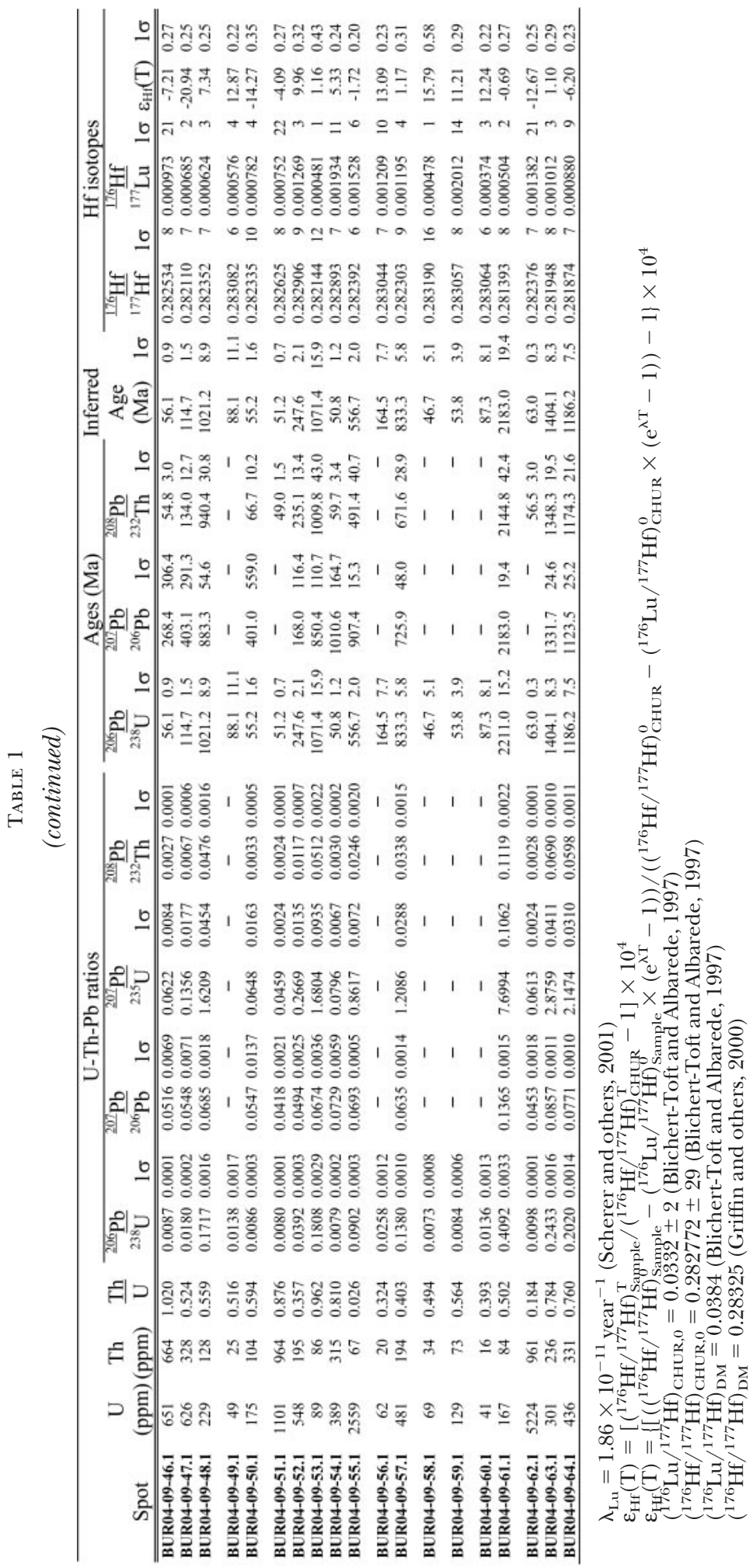




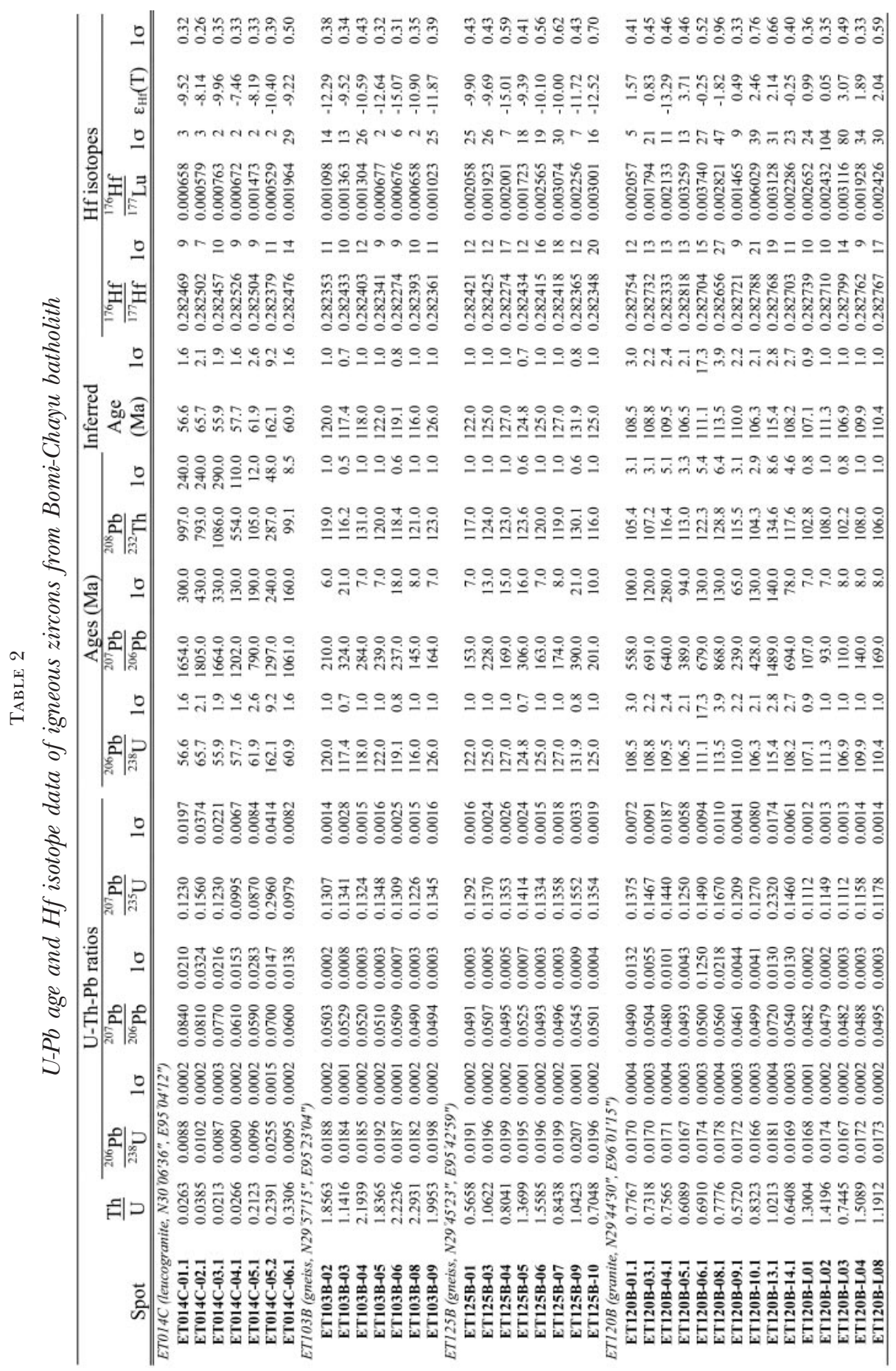




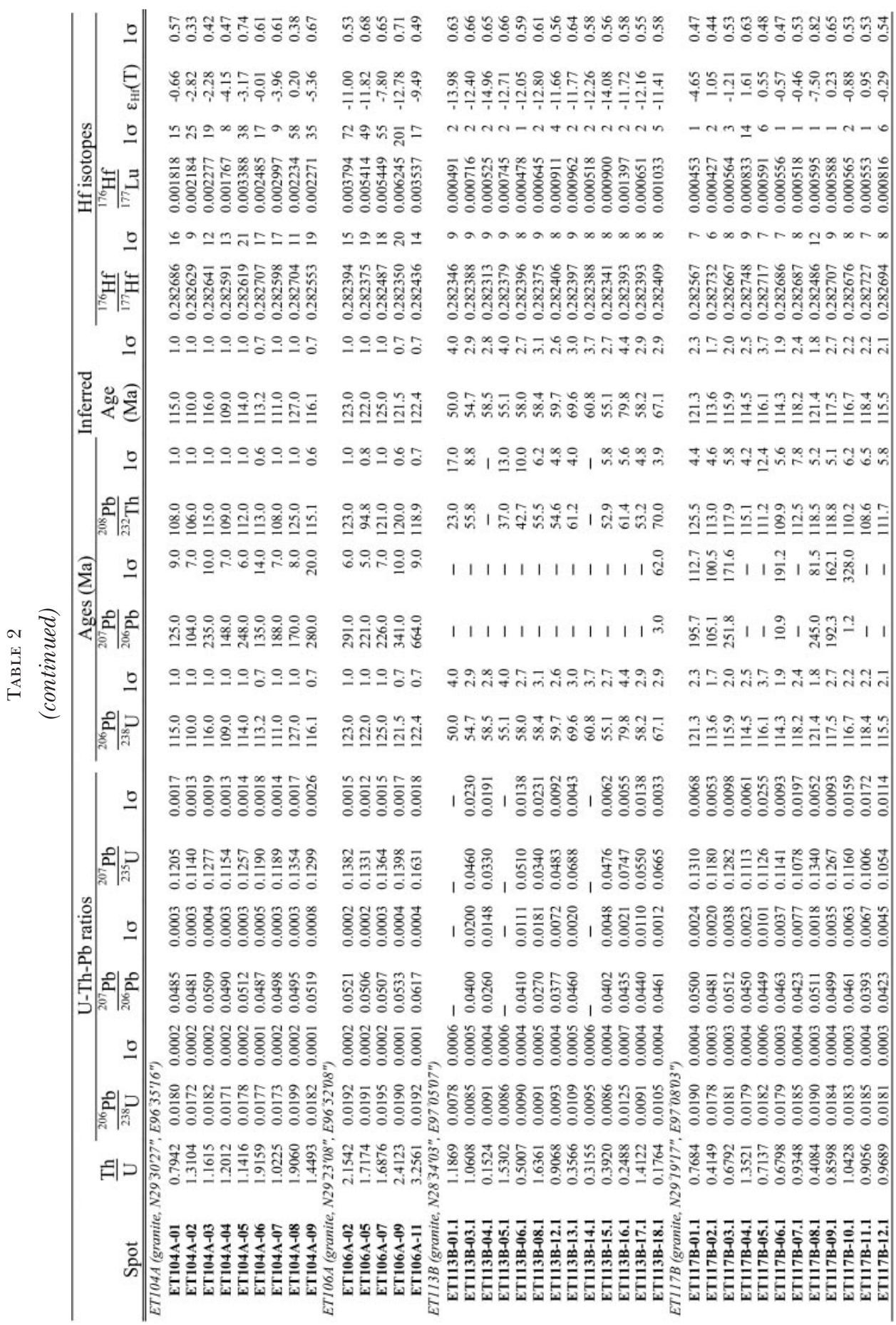




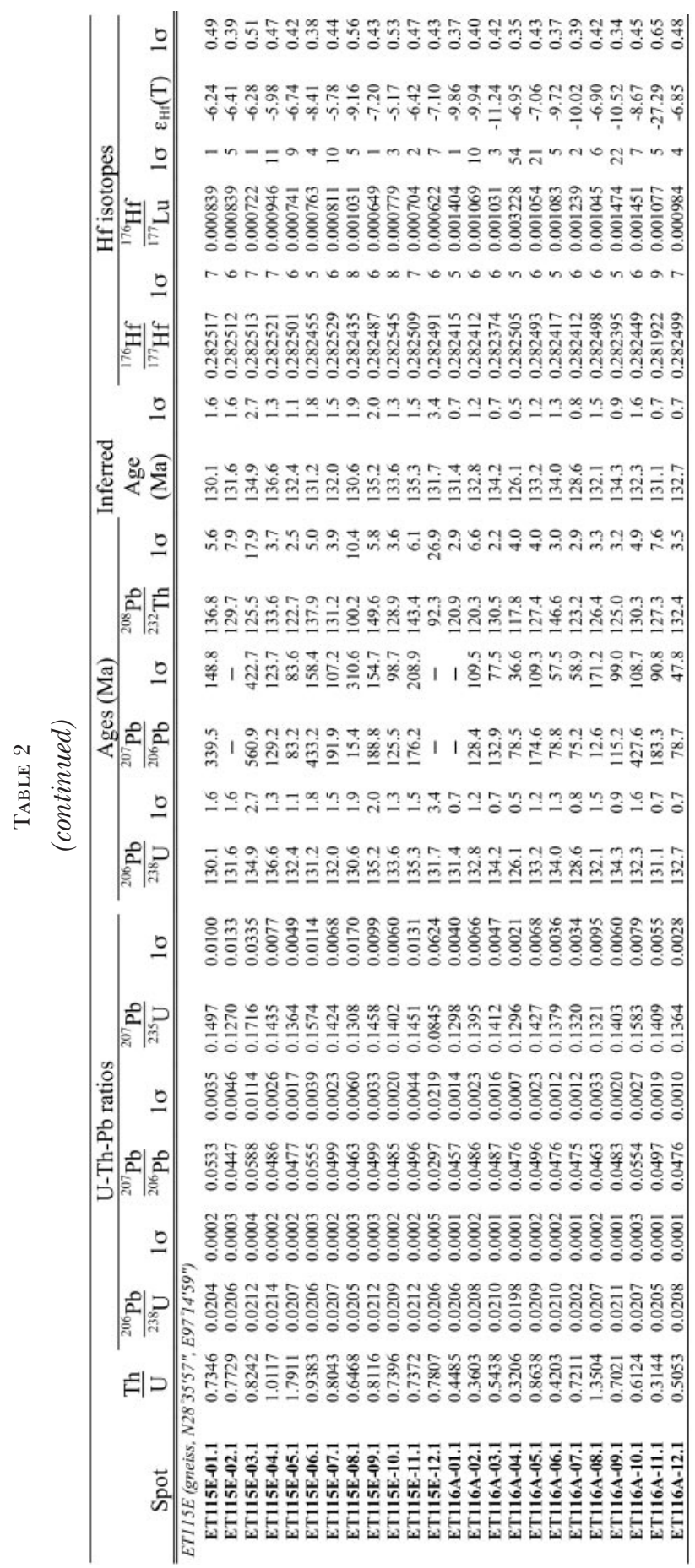


TABle 3

$\mathrm{U}-\mathrm{Pb}$ age and Hf isotope data of igneous zircons from Dianxi-Burma batholith

\begin{tabular}{|c|c|c|c|c|c|c|c|}
\hline \multirow[b]{2}{*}{ Spot } & \multirow{2}{*}{$\begin{array}{c}\text { U-Pb } \\
\text { Age }(\mathrm{Ma})^{*}\end{array}$} & \multirow[b]{2}{*}{$1 \sigma$} & \multicolumn{5}{|c|}{ Hf isotopes } \\
\hline & & & ${ }^{176} \mathrm{Hf} /{ }^{177} \mathrm{Hf}$ & $1 \sigma$ & ${ }^{176} \mathrm{Lu} /{ }^{177} \mathrm{Hf}$ & $\varepsilon_{\mathrm{HI}}(\mathrm{T})$ & $1 \sigma$ \\
\hline \multicolumn{8}{|c|}{ TCBH-6 (N25 $05^{\prime} 52^{\prime \prime}$, E98 $\left.15^{\prime} 21^{\prime \prime}\right)$} \\
\hline TCBH-6 -1 & 72.6 & 1.2 & 0.282429 & 5 & 0.000920 & -10.59 & 0.18 \\
\hline TCBH-6 -2 & 76.8 & 5.2 & 0.282442 & 6 & 0.001500 & -10.08 & 0.21 \\
\hline ТCВH-6 -3 & 68.7 & 2.2 & 0.282408 & 8 & 0.001231 & -11.44 & 0.28 \\
\hline TCBH-6 -4 & 80.6 & 3.9 & 0.282389 & 8 & 0.001578 & -11.88 & 0.27 \\
\hline TCBH-6 -5 & 72.9 & 1.1 & 0.282425 & 7 & 0.001261 & -10.74 & 0.24 \\
\hline TCBH-6 -6 & 71.2 & 1.1 & 0.282502 & 10 & 0.001892 & -8.10 & 0.34 \\
\hline TCBH-6 -7 & 67.1 & 3.5 & 0.282436 & 8 & 0.001028 & -10.46 & 0.28 \\
\hline ТCBH-6 -8 & 81.8 & 2.7 & 0.282439 & 6 & 0.001395 & -10.08 & 0.23 \\
\hline TCBH-6 -9 & 70.2 & 1.1 & 0.282437 & 7 & 0.001162 & -10.38 & 0.24 \\
\hline TCBH-6 -10 & 74.3 & 1.2 & 0.282430 & 6 & 0.001125 & -10.53 & 0.22 \\
\hline TCBH-6 -11 & 71.6 & 1.2 & 0.282425 & 5 & 0.001568 & -10.79 & 0.19 \\
\hline ТCBH-6 -12 & 72.9 & 1.3 & 0.282434 & 6 & 0.001470 & -10.44 & 0.20 \\
\hline TCBH-6 -13 & 70.8 & 1.4 & 0.282426 & 6 & 0.001921 & -10.79 & 0.22 \\
\hline ТСВH-6 -14 & 70.1 & 1.9 & 0.282433 & 5 & 0.002157 & -10.55 & 0.19 \\
\hline \multicolumn{8}{|c|}{ TCGY-3 (N25 $18^{\prime} 34^{\prime \prime}$, E98 $\left.15^{\prime} 24^{\prime \prime}\right)$} \\
\hline TCGY-3 -1 & 67.7 & 2.6 & 0.282401 & 9 & 0.001607 & -11.70 & 0.30 \\
\hline TCGY-3 -2 & 66.1 & 3.1 & 0.282486 & 7 & 0.001601 & -8.73 & 0.26 \\
\hline TCGY-3 -3 & 66.0 & 2.5 & 0.282445 & 7 & 0.002603 & -10.22 & 0.24 \\
\hline TCGY-3-4 & 69.2 & 2.5 & 0.282373 & 6 & 0.002142 & -12.68 & 0.22 \\
\hline TCGY-3 -5 & 76.4 & 3.8 & 0.282392 & 8 & 0.001362 & -11.82 & 0.30 \\
\hline TCGY-3 -6 & 69.4 & 2.5 & 0.282399 & 5 & 0.001781 & -11.76 & 0.18 \\
\hline TCGY-3 -7 & 71.6 & 2.8 & 0.282393 & 5 & 0.003719 & -12.01 & 0.18 \\
\hline TCGY-3 -8 & 68.4 & 2.5 & 0.282399 & 5 & 0.002401 & -11.81 & 0.19 \\
\hline TCGY-3 -9 & 67.6 & 2.5 & 0.282373 & 5 & 0.001789 & -12.72 & 0.17 \\
\hline TCGY-3 -10 & 66.4 & 2.5 & 0.282373 & 6 & 0.001403 & -12.74 & 0.22 \\
\hline TCGY-3 -11 & 62.4 & 2.3 & 0.282397 & 6 & 0.002329 & -12.00 & 0.21 \\
\hline TCGY-3 -12 & 66.6 & 2.5 & 0.282435 & 8 & 0.002823 & -10.58 & 0.30 \\
\hline TCGY-3 -14 & 69.7 & 2.6 & 0.282397 & 4 & 0.002317 & -11.85 & 0.15 \\
\hline \multicolumn{8}{|c|}{ TCGY11 (N25 $20^{\prime} 56^{\prime \prime}$, E98 $\left.17^{\prime} 43^{\prime \prime}\right)$} \\
\hline TCGY11 -1-1 & 75.7 & 5.9 & 0.282519 & 11 & 0.001131 & -7.37 & 0.40 \\
\hline TCGY11 -2-1 & 84.4 & 6.6 & 0.282516 & 7 & 0.001273 & -7.30 & 0.26 \\
\hline TCGY11 -3-1 & 86.5 & 3.2 & 0.282528 & 7 & 0.001290 & -6.81 & 0.25 \\
\hline TCGY11 -5-1 & 74.0 & 3.6 & 0.282504 & 9 & 0.000932 & -7.90 & 0.32 \\
\hline TCGY11 -6-1 & 67.8 & 2.7 & 0.282570 & 7 & 0.001024 & -5.72 & 0.25 \\
\hline TCGY11 -7-1 & 80.3 & 5.1 & 0.282497 & 9 & 0.001018 & -7.96 & 0.33 \\
\hline TCGY11 -8-1 & 72.3 & 2.7 & 0.282529 & 8 & 0.000916 & -7.05 & 0.28 \\
\hline TCGY11 -9-1 & 73.8 & 2.9 & 0.282469 & 9 & 0.000700 & -9.10 & 0.32 \\
\hline TCGY11 -10-1 & 74.8 & 2.8 & 0.282497 & 8 & 0.000708 & -8.11 & 0.28 \\
\hline TCGY11 -11-1 & 81.1 & 3.0 & 0.282472 & 8 & 0.000938 & -8.82 & 0.29 \\
\hline TCGY11 -12-1 & 83.3 & 5.2 & 0.282551 & 7 & 0.001415 & -6.07 & 0.25 \\
\hline TCGY11 -13-1 & 76.1 & 2.9 & 0.282597 & 8 & 0.001761 & -4.62 & 0.27 \\
\hline TCGY11 -14-1 & 74.0 & 2.7 & 0.282520 & 6 & 0.001416 & -7.38 & 0.23 \\
\hline \multicolumn{8}{|c|}{ TCLL-9 (N24 $55^{\prime} 33^{\prime \prime}$, E98 $\left.15^{\prime} 19^{\prime \prime}\right)$} \\
\hline TCLL-9 -2 & 52.9 & 1.2 & 0.282447 & 5 & 0.000905 & -10.38 & 0.19 \\
\hline TCLL-9 -3 & 55.8 & 1.5 & 0.282490 & 6 & 0.001114 & -8.79 & 0.22 \\
\hline TCLL-9 -4 & 53.4 & 1.1 & 0.282457 & 6 & 0.001666 & -10.04 & 0.23 \\
\hline TCLL-9 -5 & 52.5 & 1.3 & 0.282485 & 5 & 0.000924 & -9.05 & 0.19 \\
\hline TCLL-9 -6 & 54.6 & 0.9 & 0.282470 & 6 & 0.001500 & -9.55 & 0.20 \\
\hline TCLL-9 -7 & 54.3 & 0.8 & 0.282453 & 6 & 0.001333 & -10.15 & 0.21 \\
\hline TCLL-9 -8 & 60.0 & 0.9 & 0.282583 & 9 & 0.001576 & -5.44 & 0.32 \\
\hline TCLL-9 -9 & 48.6 & 2.3 & 0.282483 & 5 & 0.001119 & -9.20 & 0.18 \\
\hline TCLL-9 -10 & 54.6 & 1.3 & 0.282480 & 5 & 0.001355 & -9.19 & 0.19 \\
\hline TCLL-9 -11 & 51.8 & 1.0 & 0.282505 & 5 & 0.000953 & -8.34 & 0.19 \\
\hline TCLL-9 -12 & 52.8 & 0.9 & 0.282479 & 5 & 0.001356 & -9.25 & 0.19 \\
\hline TCLL-9 -13 & 52.5 & 1.1 & 0.282498 & 6 & 0.001290 & -8.60 & 0.20 \\
\hline TCLL-9 -14 & 51.2 & 1.2 & 0.282489 & 4 & 0.001493 & -8.94 & 0.15 \\
\hline TCLL-9 -15 & 52.7 & 0.9 & 0.282475 & 5 & 0.001223 & -9.38 & 0.19 \\
\hline TCLL-9 -16 & 50.6 & 0.9 & 0.282519 & 5 & 0.001724 & -7.91 & 0.18 \\
\hline
\end{tabular}


TABle 3

(continued)

\begin{tabular}{|c|c|c|c|c|c|c|c|}
\hline \multirow[b]{2}{*}{ Spot } & \multirow{2}{*}{$\begin{array}{c}\text { U-Pb } \\
\text { Age }(\mathrm{Ma})^{*}\end{array}$} & \multirow[b]{2}{*}{$1 \sigma$} & \multicolumn{5}{|c|}{ Hf isotopes } \\
\hline & & & ${ }^{176} \mathrm{Hf} /{ }^{177} \mathrm{Hf}$ & $1 \sigma$ & ${ }^{176} \mathrm{Lu} /{ }^{177} \mathrm{Hf}$ & $\varepsilon_{\mathrm{Hf}}(\mathrm{T})$ & $1 \sigma$ \\
\hline \multicolumn{8}{|c|}{ GLS-8 (N26 $00^{\prime} 50^{\prime \prime}$, E9 $\left.{ }^{\circ} 39^{\prime} 01^{\prime \prime}\right)$} \\
\hline GLS-8 -2 & 122.5 & 2.1 & 0.282487 & 7 & 0.000909 & -7.48 & 0.25 \\
\hline GLS-8 -3 & 122.5 & 2.5 & 0.282513 & 6 & 0.001055 & -6.57 & 0.22 \\
\hline GLS-8 -4 & 124.6 & 1.9 & 0.282522 & 5 & 0.001366 & -6.24 & 0.19 \\
\hline GLS-8 -5 & 124.6 & 2.0 & 0.282523 & 6 & 0.002015 & -6.25 & 0.20 \\
\hline GLS-8 -6 & 120.8 & 2.0 & 0.282395 & 6 & 0.001257 & -10.79 & 0.22 \\
\hline GLS-8 -7 & 121.7 & 2.0 & 0.282564 & 6 & 0.002890 & -4.93 & 0.23 \\
\hline GLS-8 -8 & 125.2 & 2.0 & 0.282433 & 5 & 0.000764 & -9.31 & 0.19 \\
\hline GLS-8 -9 & 117.5 & 2.1 & 0.282525 & 6 & 0.001291 & -6.26 & 0.21 \\
\hline GLS-8 -10 & 119.6 & 1.9 & 0.282526 & 6 & 0.001291 & -6.19 & 0.21 \\
\hline GLS-8 -11 & 123.2 & 1.9 & 0.282501 & 6 & 0.001036 & -6.96 & 0.22 \\
\hline GLS-8 -12 & 124.6 & 1.9 & 0.282510 & 5 & 0.001194 & -6.65 & 0.18 \\
\hline GLS-8 -13 & 122.8 & 1.9 & 0.282451 & 5 & 0.001093 & -8.77 & 0.17 \\
\hline GLS-8 -14 & 122.9 & 1.9 & 0.282520 & 5 & 0.001251 & -6.34 & 0.18 \\
\hline GLS-8 -15 & 117.0 & 2.0 & 0.282366 & 6 & 0.001250 & -11.90 & 0.23 \\
\hline \multicolumn{8}{|c|}{ GLS-36 (N27 $45^{\prime} 32^{\prime \prime}$, E9 $\left.{ }^{\circ} 35^{\prime} 07^{\prime \prime}\right)$} \\
\hline GLS-36 -1 & 116.7 & 5.1 & 0.282471 & 6 & 0.000588 & -8.15 & 0.20 \\
\hline GLS-36 -2 & 129.7 & 8.4 & 0.282506 & 5 & 0.000923 & -6.67 & 0.19 \\
\hline GLS-36 -3 & 118.3 & 4.5 & 0.282395 & 6 & 0.000599 & -10.79 & 0.20 \\
\hline GLS-36 -4 & 117.9 & 4.4 & 0.282470 & 5 & 0.000731 & -8.14 & 0.19 \\
\hline GLS-36 -5 & 124.0 & 4.6 & 0.282455 & 5 & 0.001043 & -8.59 & 0.17 \\
\hline GLS-36 -6 & 127.5 & 4.7 & 0.282503 & 4 & 0.001302 & -6.85 & 0.16 \\
\hline GLS-36 -7 & 126.7 & 4.9 & 0.282498 & 7 & 0.001466 & -7.04 & 0.26 \\
\hline GLS-36 -8 & 120.8 & 5.7 & 0.282470 & 5 & 0.000898 & -8.10 & 0.19 \\
\hline GLS-36 -9 & 127.8 & 4.9 & 0.282494 & 6 & 0.001396 & -7.16 & 0.23 \\
\hline GLS-36 -10 & 125.2 & 4.6 & 0.282470 & 5 & 0.001365 & -8.06 & 0.18 \\
\hline GLS-36 -11 & 125.0 & 4.7 & 0.282482 & 6 & 0.001447 & -7.64 & 0.20 \\
\hline GLS-36 -12 & 129.3 & 4.8 & 0.282506 & 7 & 0.001083 & -6.67 & 0.26 \\
\hline GLS-36 -13 & 126.7 & 5.1 & 0.282472 & 5 & 0.000830 & -7.91 & 0.18 \\
\hline GLS-36 -14 & 129.1 & 8.9 & 0.282552 & 11 & 0.002123 & -5.15 & 0.38 \\
\hline \multicolumn{8}{|c|}{ GLS-53 (N27 $12^{\prime} 11^{\prime \prime}$, E9 $\left.{ }^{\circ} 42^{\prime} 48^{\prime \prime}\right)$} \\
\hline GLS-53 -1 & 80.9 & 3.0 & 0.282484 & 5 & 0.001504 & -8.51 & 0.18 \\
\hline GLS-53 -2 & 76.1 & 5.1 & 0.282511 & 9 & 0.001306 & -7.63 & 0.32 \\
\hline GLS-53 -4-1 & 72.1 & 2.8 & 0.282510 & 5 & 0.001415 & -7.75 & 0.19 \\
\hline GLS-53 -5-1 & 68.5 & 2.6 & 0.282547 & 5 & 0.001517 & -6.54 & 0.17 \\
\hline GLS-53 -6-1 & 73.2 & 2.7 & 0.282491 & 8 & 0.002124 & -8.44 & 0.29 \\
\hline GLS-53 -7-2 & 83.9 & 4.4 & 0.282470 & 10 & 0.001571 & -8.93 & 0.35 \\
\hline GLS-53 -8-1 & 78.7 & 3.0 & 0.282494 & 22 & 0.001280 & -8.18 & 0.77 \\
\hline GLS-53 -9-2 & 72.9 & 7.5 & 0.282578 & 12 & 0.001418 & -5.34 & 0.43 \\
\hline GLS-53 -10-1 & 79.9 & 3.0 & 0.282527 & 5 & 0.001336 & -7.00 & 0.18 \\
\hline GLS-53 -11-1 & 65.8 & 2.5 & 0.282490 & 4 & 0.001159 & -8.58 & 0.15 \\
\hline GLS-53 -12-1 & 75.3 & 4.8 & 0.282574 & 4 & 0.001385 & -5.42 & 0.15 \\
\hline GLS-53 -13-1 & 63.6 & 4.0 & 0.282508 & 5 & 0.001278 & -7.99 & 0.17 \\
\hline \multicolumn{8}{|c|}{ GLS-62 (N27 $08^{\prime} 01^{\prime \prime}$, E98 $\left.49^{\prime} 37^{\prime \prime}\right)$} \\
\hline GLS-62 -1 & 117.1 & 1.8 & 0.282583 & 8 & 0.000943 & -4.19 & 0.30 \\
\hline GLS-62 -2 & 120.9 & 3.3 & 0.282574 & 6 & 0.001198 & -4.46 & 0.23 \\
\hline GLS-62 -3 & 126.2 & 1.8 & 0.282596 & 5 & 0.001051 & -3.56 & 0.19 \\
\hline GLS-62 -4 & 116.1 & 1.9 & 0.282613 & 6 & 0.001433 & -3.19 & 0.22 \\
\hline GLS-62 -5 & 116.2 & 1.8 & 0.282578 & 7 & 0.001059 & -4.39 & 0.26 \\
\hline GLS-62 -6 & 136.8 & 2.0 & 0.282761 & 5 & 0.003818 & 2.25 & 0.17 \\
\hline GLS-62 -7 & 124.7 & 1.9 & 0.282625 & 6 & 0.001618 & -2.62 & 0.22 \\
\hline GLS-62 -8 & 109.0 & 2.7 & 0.282605 & 7 & 0.001394 & -3.62 & 0.26 \\
\hline GLS-62 -9 & 133.6 & 2.0 & 0.282618 & 6 & 0.001376 & -2.64 & 0.22 \\
\hline GLS-62 -10 & 110.7 & 1.9 & 0.282603 & 6 & 0.001504 & -3.67 & 0.21 \\
\hline GLS-62 -11 & 118.4 & 2.2 & 0.282721 & 7 & 0.002695 & 0.59 & 0.24 \\
\hline GLS-62 -12 & 135.9 & 2.2 & 0.282613 & 6 & 0.001173 & -2.77 & 0.22 \\
\hline GLS-62 -13 & 118.7 & 1.8 & 0.282587 & 6 & 0.001077 & -4.05 & 0.20 \\
\hline GLS-62 -14 & 126.7 & 4.9 & 0.282629 & 5 & 0.001530 & -2.41 & 0.18 \\
\hline GLS-62 -15 & 112.3 & 1.8 & 0.282652 & 12 & 0.003652 & -2.07 & 0.41 \\
\hline
\end{tabular}

${ }^{* 206} \mathrm{~Pb} /{ }^{238} \mathrm{U}$ Ages (Yang and others, 2006) 


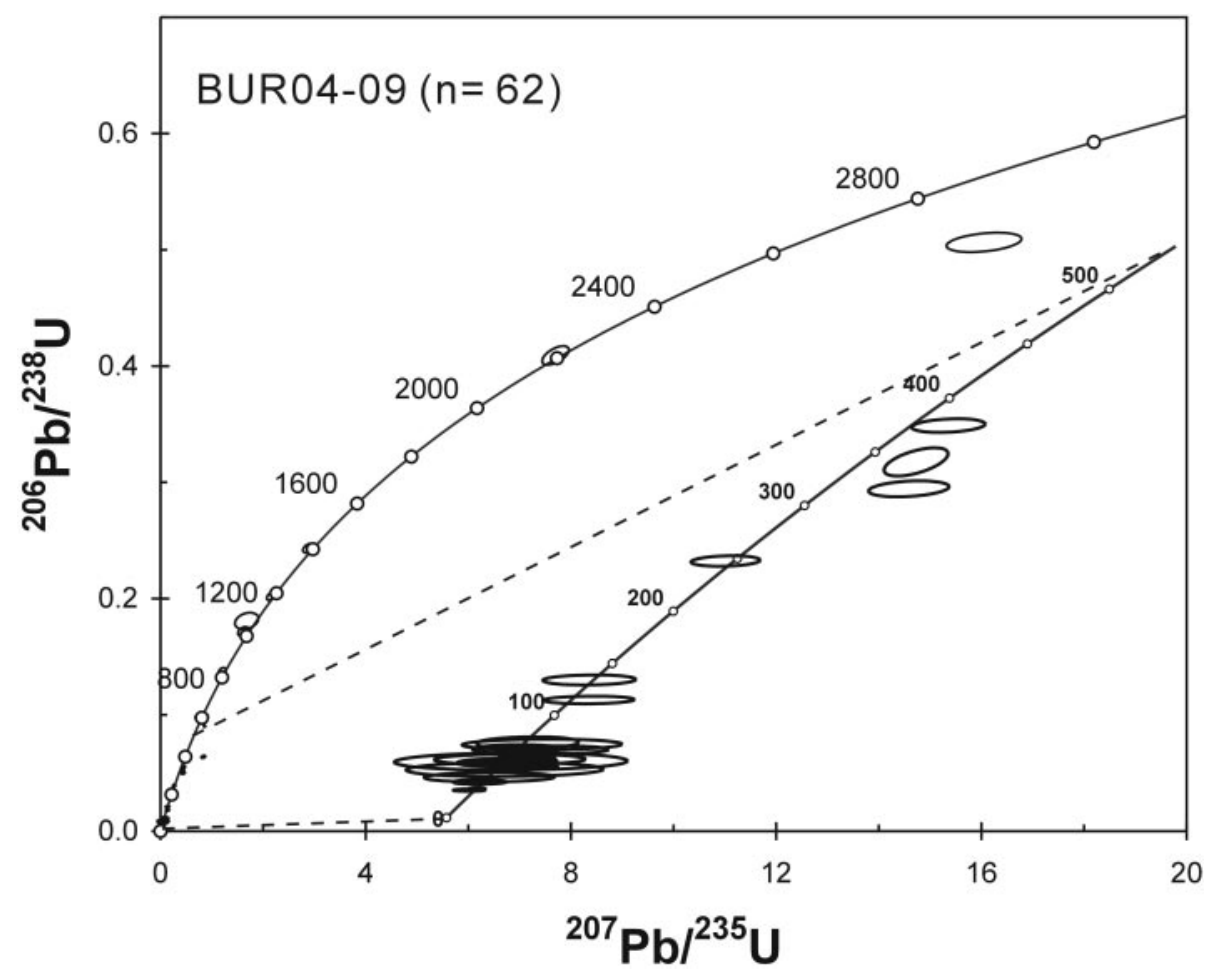

Fig. 2. Concordia diagram of zircon U-Pb results of sandstone sample BUR04-09 from the uppermost Pegu Group, the Inner-Burma Tertiary Basin.

extension of the Transhimalayan plutons (Searle and others, 1987; Chung and others, 2005; and references therein). In the Lhasa terrane, the plutonic bodies are generally divided into two suites, that is, a southern Gangdese belt that comprises essentially Late Cretaceous and Paleogene rocks marked with I-type petrochemical compositions and a northern plutonic belt that is dominated by Cretaceous peraluminous, or S-type, granitoids (Pan and others, 2004). Consequently, rocks from these two belts differ remarkably in their $\mathrm{Sr}$ and $\mathrm{Nd}$ isotope ratios (Wen, ms, 2007). Zircons can effectively preserve the initial Hf isotope compositions of the host magmas (Griffin and others, 2002), thus allowing their Hf isotope ratios to be utilized in much the same way as whole-rock $\mathrm{Nd}$ isotopes have been used as a powerful geochemical tracer. Magmatic zircons from the two plutonic belts in the Lhasa terrane, therefore, display distinguishable Hf isotope signatures, that is, the Gangdese ones show high, positive $\varepsilon_{\mathrm{Hf}}(\mathrm{T})$ values indicative of a prominent juvenile mantle contribution in the petrogenesis and those from granitoids of the northern suite are overwhelmed by negative, low $\varepsilon_{\mathrm{Hf}}(\mathrm{T})$ values that yield Paleoproterozoic model ages and suggest old continental crust sources $(\mathrm{Chu}$, ms, 2006; Chu and others, 2006).

Our zircon Hf isotope results from the Bomi-Chayu and Dianxi-Burma batholiths, as described above, do not resemble the Gangdese data but are rather similar to those of the northern plutons. This is consistent with the whole-rock isotopic correlation that, for instance, granitoids from both Bomi-Chayu (Lin, ms, 2007) and Dianxi-Burma batholiths (Chen and others, 2001; Yang and others, 2006; Mitchell and others, 2007) commonly exhibit negative $\varepsilon_{\mathrm{Nd}}(\mathrm{T})$ values from -2 to -12 , comparable to the range of the northern plutonic rocks (Harris and others, 1990; Wen, ms, 2007) and different 

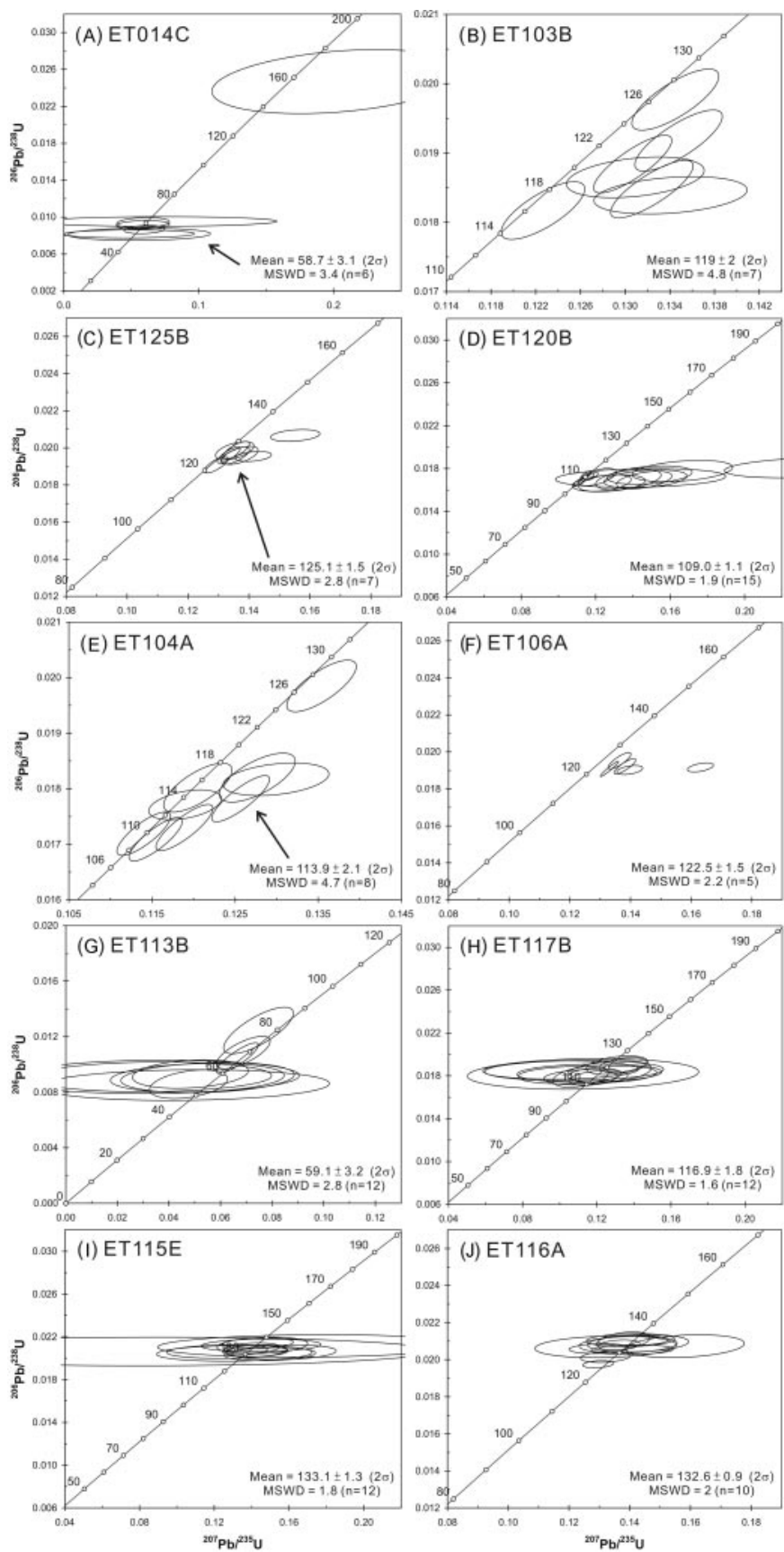

Fig. 3. Concordia diagrams of zircon U-Pb results of granitoids from the Bomi-Chayu batholith.

from the positive Gangdese $\varepsilon_{\mathrm{Nd}}(\mathrm{T})$ values (Wen and others, 2005; Wen, ms, 2007). The distinctive Hf isotope feature of igneous zircons from the Gangdese batholith is illustrated using figure 4 , in which 146 out of 168 total analyses $(\sim 87 \%)$ demonstrate 


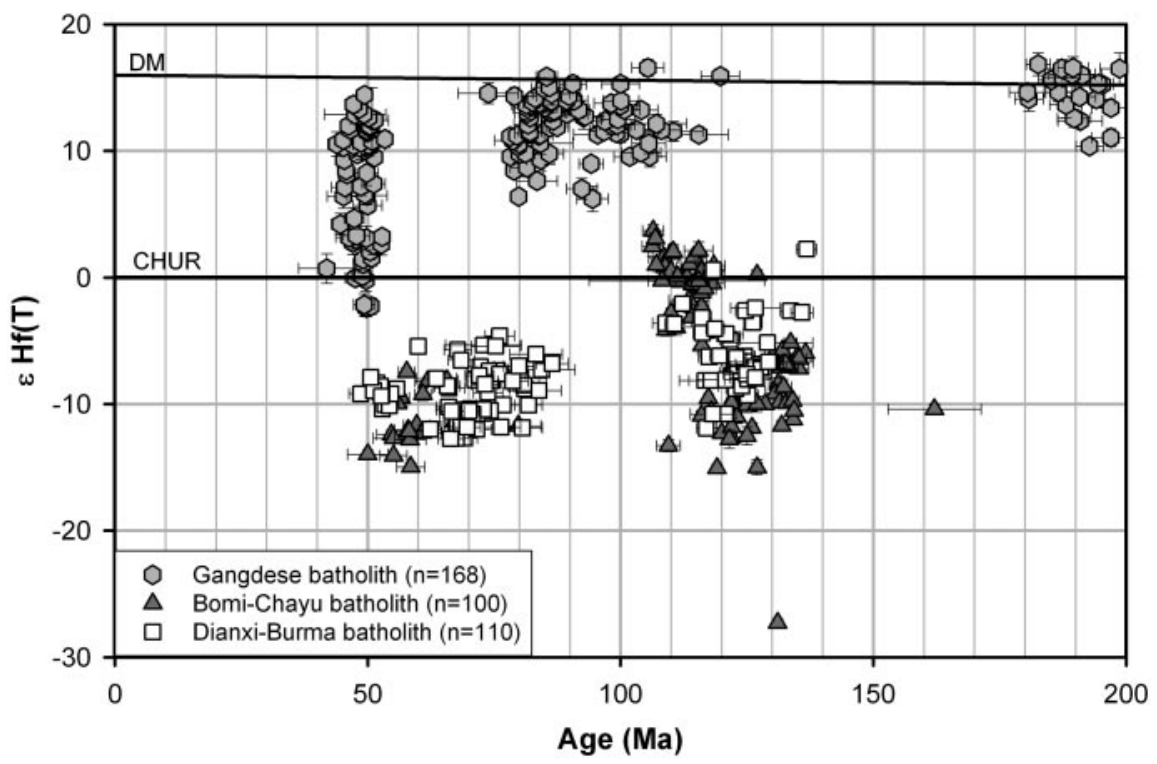

Fig. 4. Plots of initial epsilon Hf values $\left[\varepsilon_{\mathrm{Hf}}(\mathrm{T})\right]$ versus U-Pb ages of magmatic zircons from the Bomi-Chayu and Dianxi-Burma batholiths. The Gangdese data (Chu, ms, 2006; Chu and others, 2006) are also plotted for comparison.

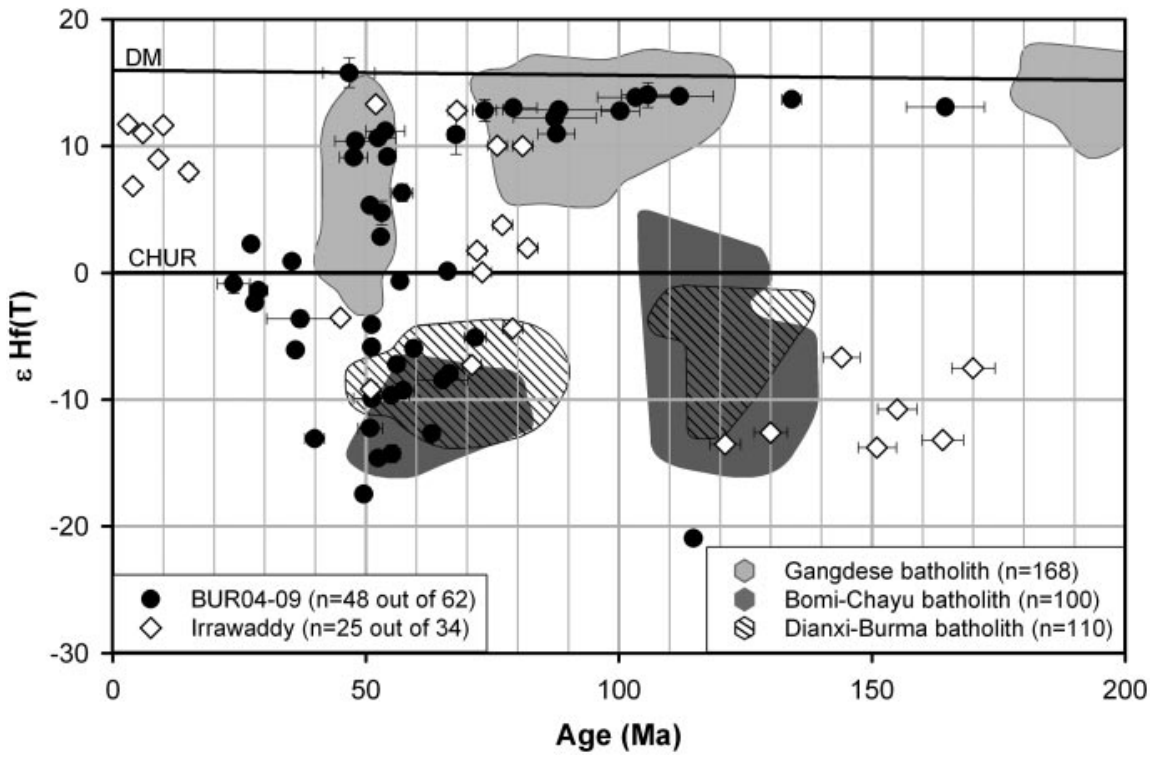

Fig. 5. Plots of $\varepsilon_{\mathrm{Hf}}(\mathrm{T})$ values vs. U-Pb ages for detrital zircons from sample BUR04-09 (this study) and Irrawaddy riverbank sediment (Bodet and Schärer, 2000). Only zircons $\leq 200 \mathrm{Ma}$ are plotted, with $1 \sigma$ analytical errors when they are larger than the symbols. See table 1 for remaining data of $>200$ Ma zircons, and for notations of $\varepsilon_{\mathrm{Hf}}(\mathrm{T})$, and chondritic uniform reservoir (CHUR) and depleted-mantle (DM) curves. The batholithic zircon fields of the 3 major Transhimalayan plutons are constructed from the previous figure. 


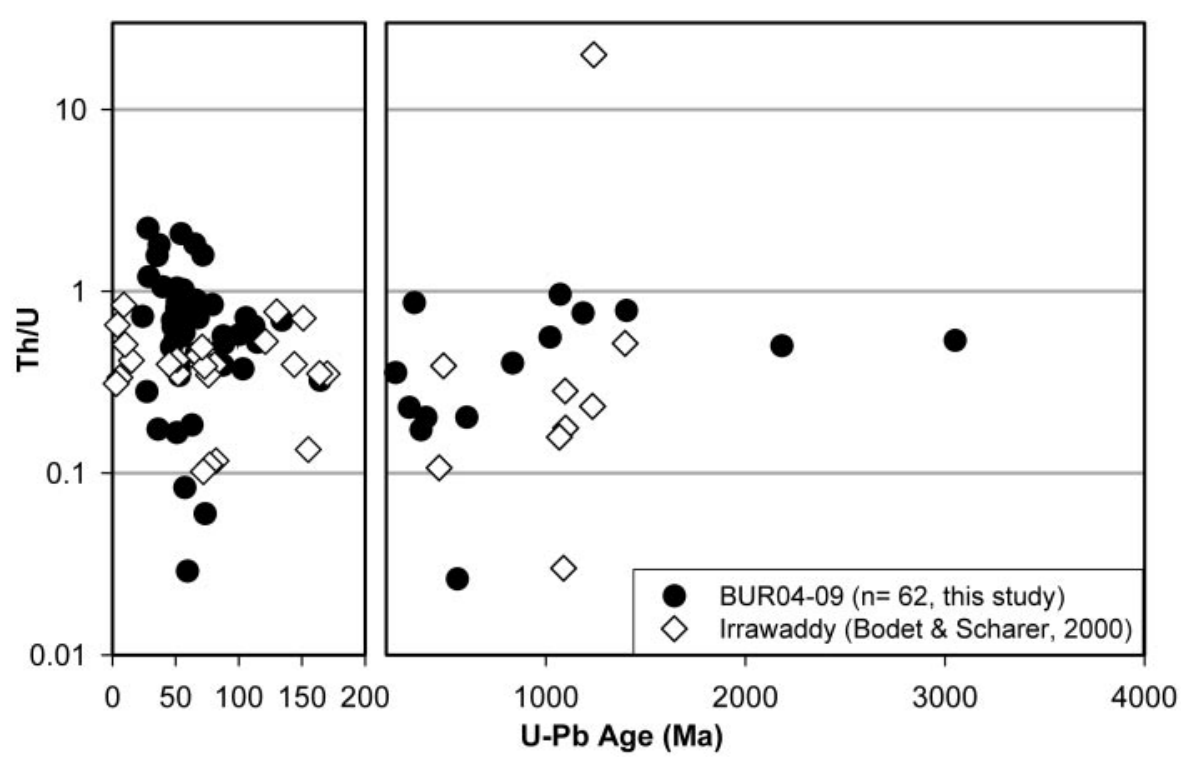

Fig. 6. Plots of $\mathrm{Th} / \mathrm{U}$ elemental ratios versus $\mathrm{U}-\mathrm{Pb}$ ages of the Burmese detrital zircons, including sample BUR04-09 of this study and riverbank sediment data reported by Bodet and Schärer (2000).

$\varepsilon_{\mathrm{Hf}}(\mathrm{T})$ values $\geq+5$ (Chu, ms, 2006; Chu and others, 2006). Such high values, in particular those with $\varepsilon_{\mathrm{Hf}}(\mathrm{T})>+10$ or even up to the depleted mantle value, have never been observed to date in zircons from any other eastern Transhimalayan plutons. Thus, this Gangdese "signal" may be used as a unique and most powerful proxy that allows "fingerprinting" detrital zircons deposited in the downstream basins for studying the sedimentary source to sink relation affiliated with the drainage evolution around the eastern Himalayan syntaxis.

\section{Interpretation of Our Inner-Burma Basin Data}

With the understanding that the current data are far from enough and furthermore detailed sampling from the studied mountainous region could produce other results, we here summarize all available zircon Hf isotope data to put forth the best interpretation on the sedimentary source to sink relation. We assume the Gangdese batholith to be a unique Transhimalayan complex that, according to Chung and others (2007), situates in an "oceanic" domain in the Gondwana-derived Lhasa terrane. It may be correlated to the specific part of the eastern Lachlan Fold Belt in Australia resulting from accretion of an Ordovician intraoceanic arc complex to this part of east Gondwana (Glen and others, 1998; Collins and Hobbs, 2001).

In figure 5, all 48 detrital zircons of $<200$ Ma ages from the sample BUR04-09 are considered magmatic in origin because most of these zircons show euhedral to subhedral morphologies and all but three grains have $\mathrm{Th} / \mathrm{U}$ ratios $>0.1$ (fig. 6). Large plutons that crop out around the eastern Himalayas, that is, the Dianxi-Burma and Bomi-Chayu batholiths that are cut through by the Irrawaddy drainage (fig. 1), are likely the principal source rocks. The zircon Hf isotope data, however, indicate that these nearby batholiths might supply zircons only with negative $\varepsilon_{\mathrm{Hf}}(\mathrm{T})$ values (fig. 5). Abundant zircons that exhibit positive $\varepsilon_{\mathrm{Hf}}(\mathrm{T})$ values, in particular those showing $\varepsilon_{\mathrm{Hf}}(\mathrm{T}) \geq+10$ (fig. 5), could only be possibly sourced from the Gangdese batholith. Overall, sample BUR04-09 contains 24 zircons (1 of Jurassic, 11 of Cretaceous and 12 of 
Paleogene ages) that display positive $\varepsilon_{\mathrm{Hf}}(\mathrm{T})$ values and signal the Gangdese "fingerprint", if taking the chondritic Hf isotope line, $\varepsilon_{\mathrm{Hf}}(\mathrm{T})=0$, as a general divide for the Gangdese and the other two batholiths (fig. 5). Even if counting only those with $\varepsilon_{\mathrm{Hf}}(\mathrm{T}) \geq+10$, there are still 16 zircon grains that show the Gangdese "fingerprint".

The high abundance of Gangdese-derived zircons, that is, 24 out of a sum of 62 grains analyzed $(\sim 40 \%)$, or, at minimal, 16 grains out of them $(\sim 26 \%)$, in sample BUR04-09 implies a source-sink link and thus suggests major mountain river(s) from southeastern Tibet to have drained into the Inner-Burma Basin. This evidence lends firm support to the topographic argument that the Yarlu Tsangpo, now flowing past the southern margin of the Gangdese batholith (fig. 1), was in the upper reaches of the Irrawaddy drainage (Clark and others, 2004). Given that our sample is from the uppermost Pegu Group of Late Miocene depositional age, an additional important inference would be that by the time the ancestral Yarlu Tsangpo should have started draining into the Irrawaddy. This correlation is in accord with the timing of dextral movement along the Jiali fault system (fig. 1), most active during ca. 18 and $12 \mathrm{Ma}$ in the Middle Miocene (Lee and others, 2003), through which the courses from the Yarlu Tsangpo to the Irrawaddy River may have connected together (see below for more discussion).

In sample BUR04-09, the remaining 23 grains of $<200$ Ma zircon that show negative $\varepsilon_{\mathrm{Hf}}(\mathrm{T})$ values include 22 grains dated from ca. 70 to $24 \mathrm{Ma}$ (fig. 5). Whereas grains of 70 to $50 \mathrm{Ma}$ ages appear most likely supplied from the neighboring Dianxi-Burma and/or Bomi-Chayu batholiths, those 40 to $24 \mathrm{Ma}$ ones plot outside and do not match any fields so imply additional source(s). We suspect this to be the Late Oligocene-Early Miocene (ca. 35-23 Ma) syntectonic granites emplaced in the Mogok metamorphic belt (Barley and others, 2003; Searle and others, 2007). Up to now, however, zircon Hf isotope data are unavailable from these potential source rocks for direct comparison.

\section{New Interpretation for Existing Data}

A Gangdese connection can also account for the Irrawaddy "anomaly" previously attributed to "large proportion of mantle derived magmas" (Bodet and Schärer, 2000). As we have shown in figure 5, 25 out of 34 analyses of detrital zircons in the Irrawaddy riverbank sediment yielded $\mathrm{U}-\mathrm{Pb}$ ages $<200 \mathrm{Ma}$. These younger zircons, all with $\mathrm{Th} / \mathrm{U}$ ratios $>0.1$, include 4 Late Cretaceous-Paleogene grains with high, positive $\varepsilon_{\mathrm{Hf}}(\mathrm{T})$ values $\geq+10$ that signal the Gangdese "fingerprint" and 3 coeval grains with negative $\varepsilon_{\mathrm{Hf}}(\mathrm{T})$ values that plot in the range of the Dianxi-Burma/Bomi-Chayu data. There are 4 grains, dated around 70 to $80 \mathrm{Ma}$ (fig. 5), with positive $\varepsilon_{\mathrm{Hf}}(\mathrm{T})$ values that plot slightly above the chondritic line but outside the data fields of the known possible source rocks, which makes it difficult to determine their sources. Moreover, 7 grains aged between ca. 170 and 120 Ma exhibit negative $\varepsilon_{\mathrm{Hf}}(\mathrm{T})$ values that correspond to those of the Early Cretaceous Bomi-Chayu and Dianxi-Burma magmatic zircons (fig. 5). The occurrence of these older detrital zircons in the modern sediment may be attributed to local lithologies, for example, the granitic orthogneiss bodies that crop out near Mandalay city close to the Mogok metamorphic belt (fig. 1), from which abundant Jurassic zircons of ca. 170 Ma have been reported (Barley and others, 2003). This observation further implies that emplacement of the Dianxi-Burma batholith may have started during the Jurassic, but either the Jurassic plutonism occurred in a restricted area or the Jurassic plutons had not been exposed until post-Miocene time. Zircons of this age are not observed in the Upper Miocene Pegu sandstone from the Inner-Burma Basin. Such a local lithologic control may also be the cause of the specific group of 6 young zircons $\left(<15 \mathrm{Ma}\right.$ ) characterized by high $\varepsilon_{\mathrm{Hf}}(\mathrm{T})$ values (fig. 5), which we explain to be sourced from the nearby Late Miocene to Quaternary volcanoes or volcanic fields (fig. 1) that consist of basaltic to rhyodacitic rocks (Bender, 1983; Mitchell, 1993). 
Geochemical and Sr-Nd isotope data available so far for these rocks suggest an asthenospheric mantle source involved in the magma generation (Stephenson and Marshall, 1984; Maury and others, 2004; Chung, unpublished data).

Whereas these Jurassic and Miocene-Quaternary zircons could have been supplied directly by the neighboring igneous outcrops, the 4 grains of Gangdese-sourced zircons identified also in the Irrawaddy riverbank sediment cannot be the first-cycle detritus but should represent recycled materials because the Yarlu Tsangpo is now draining into the Brahmaputra River. The change of the drainage and source provenance is evidenced by comparing the detrital zircon records in our sample and the modern Irrawaddy sediment. The percentage of the Gangdese zircons, for example, drops significantly from $\sim 40$ percent ( 24 out of 62 grains) to $\sim 12$ percent ( 4 out of 34 grains). Such a drop, compensated with the addition of new detritus from local supplies, records not only the reorganization of the drainages but also the power of these mountain rivers. The Miocene connection of the Yarlu Tsangpo to the Irrawaddy had eroded large amounts of detritus from the source region in southern Tibet and transported them to the distant downstream sedimentary basins thus forming the $>10 \mathrm{~km}$ thick deposition in the Irrawaddy Delta (Bender, 1983). Loosing such power and "water head" from the plateau, which happened as soon as the Yarlu Tsangpo rerouted (see below), the Irrawaddy became a steady alluvial river (compare Schumn and others, 2000) that can merely recycle the preexisting riverbank sediments with mild erosion from exposed rocks close-by.

\section{Tectonic Forcing on the River Evolution}

Adopting the scenarios proposed for the drainage system development in Southeast Asia by Clark and others (2004) and Clift and others (2004, 2006b), combined with the knowledge of regional tectonic activities, we here summarize the evolutionary relation between the Irrawaddy and Yarlu Tsangpo through time. Before the Miocene, the Yarlu Tsangpo was one of the principal Tibetan tributaries to a single, southeastward flowing river system or the so-called "paleo-Red River" that drained into the South China Sea (fig. 7A). Although it remains uncertain about when exactly the Yarlu Tsangpo started connecting to the "paleo-Red River", a few grains of young detrital zircons ( $\leq 100 \mathrm{Ma}$ ) from the Red River drainage reported in Bodet and Schärer (2000) do reveal Gangdese like Hf isotopic signatures. Given that the present course of the Red River well follows the fault zone, we speculate the connection to have been closely associated with, if not facilitated by, initiation of the Red River and Jiali shear zones that both appear to have begun from Late Oligocene time, say, ca. 30 to $25 \mathrm{Ma}$ (Wang and others, 1998; Ding and others, 2001; Gilley and others, 2003; Lin and others, 2005), with an understanding that topographic control related to Tibetan uplift is essential in the development of mountain rivers (Schumn and others, 2000; Clift and others, 2004). Under this framework, even in the case that the "paleo-Red River" was preexisting as the conditions proposed by Clark and others (2004) or Clift and others (2004), its route would have been further modified and confined by the left-lateral movement of the Red River shear zone, most active in the Miocene (Wang and others, 1998; Searle, 2006). A better knowledge on this regard may be achieved by combined analyses of $\mathrm{U}-\mathrm{Pb}$ and $\mathrm{Hf}$ isotopes of detrital zircons from not only the riverbank but, more importantly here, Miocene to Paleogene sediments in the Red River drainage system.

Then, controlled by the Tibetan uplift and resultant change in regional topography, the "paleo-Red River" gradually lost its drainage to the neighboring river systems (Clift and others, 2006b). This drainage change, marked with river truncation in the north and river capture in the south (Brookfield, 1998), may have started from the capture of the Yarlu Tsangpo system by the Irrawaddy (fig. 7B) occurring no later than Late Miocene time when the uppermost Pegu Group was being deposited in the 


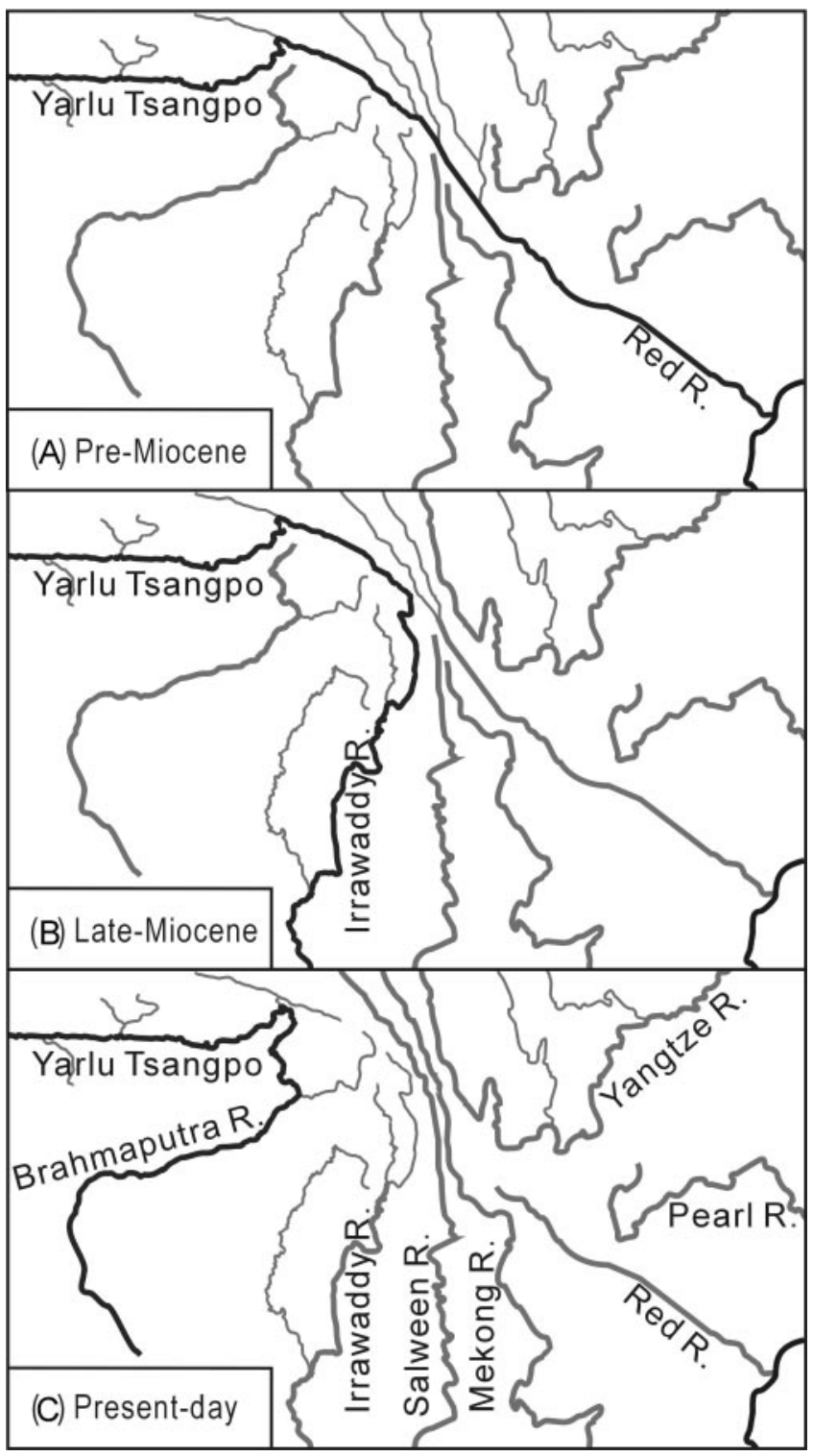

Fig. 7. Sequential change of the Yarlu Tsangpo draining direction from (A) Pre-Miocene, through (B) Late Miocene, to (C) Present-day, illustrated using the modern drainages in Southeast Asia without reconstruction for the tectonic deformation through time (after Clark and others, 2004).

Inner-Burma Tertiary Basin. Whereas regional topographic gradient control was essential, we envision such a river rerouting to have also been affiliated with, and facilitated by, the Middle Miocene (ca. 18-12 Ma) right-lateral activity of the Jiali fault (Lee and others, 2003), which has since accompanied the Gaoligong and Sagaing fault zones in the south (fig. 1) to form a regional dextral deformation system that could have not only connected the Yarlu Tsangpo and Irrawaddy drainages at the time but also accounted for the beginning and prolonged history of the clockwise rotation of Tibetan extrusion around the eastern Himalayan syntaxis (Lee and others, 2003; Lin 
and others, 2005). The link of river capturing with development of principal structures, that is, the strike-slip fault systems, in this part of the Himalayan-Tibetan orogen demonstrates the importance of tectonic control on routing mountain rivers.

Lastly, associated with the formation of the $180^{\circ}$ Big Bend gorge that cuts through the Namche Barwa Syntaxis in eastern Himalayas (fig. 1), the Yarlu Tsangpo drainage was eventually captured by the Brahmaputra River in the south (fig. 7C). Although precise age of this capture event is still unknown, it has been generally related to the sudden uplift of the Namche Barwa Syntaxis, which took place at ca. $4 \mathrm{Ma}$ (Burg and others, 1998). According to the "tectonic aneurysm" hypothesis that correlates big rivers and high mountains with a positive feedback between rapid focused erosion and upward advection of lower crust (Zeitler and others, 2001), the Namche Barwa uplift may have resulted from intensified headward erosion of the Brahmaputra River that consequently captured the Yarlu Tsangpo from the Irrawaddy to form the modern eastern Himalayan river system and gave rise to reorganization of the drainage and discharge patterns in Southeast Asia.

\section{ACKNOWLEDGMENTS}

We thank C. C. Szu and J. Q. Ji for assistance in field works, H. Tao, B. Song and L. W. Xie for help with zircon isotope analyses, M. F. Chu, T. Y. Lee and A. Mitchell for discussion, and M. Searle, Y. Najman, P. Clift, J. Aitchison and P. Zeitler for review comments that helped significantly to improve the presentation. This study benefited from financial supports by the National Science Council, Taiwan, ROC.

\section{REFERENCES}

Barley, M. E., Pickard, A. L., Zaw, K., Rak, P., and Doyle, M. G., 2003, Jurassic to Miocene magmatism and metamorphism in the Mogok metamorphic belt and the India-Eurasia collision in Myanmar: Tectonics, v. 22, n. 3, p. 1019, doi:10.1029/2002TC001398.

Bender, F., 1983, Geology of Burma: Berlin-Stuttgart, Gebrüder Borntraeger, 293 p.

Blichert-Toft, J., and Albarede, F., 1997, The Lu-Hf isotope geochemistry of chondrites and the evolution of the mantle-crust system: Earth and Planetary Science Letters, v. 148, p. 243-258.

Bodet, F., and Schärer, U., 2000, Evolution of the SE-Asian continent from U-Pb and Hf isotopes in single grains of zircon and baddeleyite from large rivers: Geochimica et Cosmochimica Acta, v. 64, p. 20672091.

Brookfield, M. E., 1998, The evolution of the great river systems of southern Asia during the Cenozoic India-Asia collision: Rivers draining southward: Geomorphology, v. 22, p. 285-312.

Burg, J. P., Nievergelt, P., Oberli, F., Seward, D., Davy, P., Maurin, J. C., Diao, Z., and Meier, M., 1998, The Nambshe-Barwa syntaxis: Evidence for exhumation related to compressional crustal folding: Journal of Asian Earth Sciences, v. 16, p. 239-252.

Campbell, I. H., Reiners, P. W., Allen, C. M., Nicolescu, S., and Upadhyay, R., 2005, He-Pb double dating of detrital zircons from the Ganges and Indus Rivers: Implication for quantifying sediment recycling and provenance studies: Earth and Planetary Science Letters, v. 237, p. 402-432.

Chen, F., Satir, M., Ji, J., and Zhong, D., 2001, Nd-Sr-Pb isotopes of Tengchong Cenozoic volcanic rocks from western Yunnan, China: Evidence for an enriched mantle source: Journal of Asian Earth Sciences, v. 21, p. $39-45$.

Chu, M. F., ms, 2006, Application of ICP-MS to the study of Transhimalayan petrogenesis: Taipei, Taiwan, National Taiwan University, Ph. D. thesis.

Chu, M. F., Chung, S. L., Song, B., Liu, D., O’Reilly, S. Y., Pearson, N. J., Ji, J. Q., and Wen, D. J., 2006, Zircon $\mathrm{U}-\mathrm{Pb}$ and $\mathrm{Hf}$ isotope constraints on the Mesozoic tectonics and crustal evolution of southern Tibet: Geology, v. 34, p. 745-748.

Chung, S. L., Chu, M. F., Zhang, Y., Xie, Y., Lo, C. H., Lee, T. Y., Lan, C. Y., Li, X., Zhang, Q., and Wang, Y., 2005, Tibetan tectonic evolution inferred from spatial and temporal variations in post-collisional magmatism: Earth-Science Reviews, v. 68, p. 173-196.

Chung, S. L., Chu, M. F., O’Reilly, S. Y., Pearson, N. J., Liu, D., Song, B., Ji, J., Lo, C. H., and Lee, T. Y., 2007, A Magmatic Perspective and Zircon Hf Isotope Constraints on Tibetan Orogenesis: Eos Transactions, AGU, 88(52), Fall Meeting Abstract T23G-05.

Clark, M. K., Schoenbohm, L. M., Royden, L. H., Whipple, K. X., Burchfiel, B. C., Zhang, X., Tang, W., Wang, E., and Chen, L., 2004, Surface uplift, tectonics, and erosion of eastern Tibet from large-scale drainage patterns: Tectonics, v. 23, TC1006, doi:10.1029/2002TC001402.

Clift, P. D., 2006, Controls on the erosion of Cenozoic Asia and the flux of clastic sediment to the ocean: Earth and Planetary Science Letters, v. 241, p. 571-580.

Clift, P. D., Layne, G. D., and Blusztajn, J., 2004, The erosional record of Tibetan uplift in the East Asian marginal seas, in Clift, P. D., Wang, P., Hayes, D., and Kuhnt, W., editors, Continent-Ocean Interactions 
in the East Asian Marginal Seas: American Geophysical Union, Geophysical Monograph Series 149, p. 255-282.

Clift, P. D., Carter, A., Campbell, I. H., Pringle, M. S., Van Lap, N., Allen, C. M., Hodges, K. V., and Tan, M. T., 2006a, Thermochronology of mineral grains in the Red and Mekong Rivers, Vietnam: Provenance and exhumation implications for Southeast Asia: Geochemistry, Geophysics and Geosystem, v. 7, Q10005, doi:10.1029/2006GC001336.

Clift, P. D., Blusztajn, J., and Nguyen, A., D., 2006b, Large-scale drainage capture and surface uplift in eastern Tibet-SW China before 24 Ma inferred from sediments of the Hanoi Basin, Vietnam: Geophysical Research Letters, v. 33, L19403, doi:10.1029/2006GL027772.

Collins, W. J., and Hobbs, B. E., 2001, What caused the Early Silurian change from mafic to silicic (S-type) magmatism in the eastern Lachlan Fold Belt?: Australian Journal of Earth Sciences, v. 48, p. 25-41.

Ding, L., Zhong, D., Yin, A., Kapp, P., and Harrison, P. M., 2001, Cenozoic structural and metamorphic evolution of the eastern Himalayan syntaxis (Namche Barwa): Earth and Planetary Science Letters, v. 192 , p. $423-438$.

Finlayson, D. P., Montgomery, D. R., and Hallet, B., 2002, Spatial coincidence of rapid inferred erosion with young metamorphic massifs in the Himalayas: Geology, v. 30, p. 219-222.

Gilley, L. D., Harrison, T. M., Leloup, P. H., Ryerson, F. J., Lovera, O. M., and Wang, J. H., Direct dating of left-lateral deformation along the Red River shear zone, China and Vietnam: Journal of Geophysical Research, v. 108, No. B2, 2127, doi:10.1029/2001JB001726.

Glen, R. A., Walshe, J. L., Barron, L. M., and Watkins, J. J., 1998, Ordovician convergent-margin volcanism and tectonism in the Lachlan sector of east Gondwana: Geology, v. 26, p. 751-754.

Griffin, W. L., Wang, X., Jackson, S. E., Pearson, N. J., O'Reilly, S. Y, Xu, X., and Zhou, X., 2002, Zircon chemistry and magma mixing, SE China: In-situ analysis of Hf isotopes, Tonglu and Pingtan igneous complexes: Lithos, v. 61, p. 237-269.

Hallet, B., and Molnar, P., 2001, Distorted drainage basins as markers of crustal strain east of the Himalaya: Journal of Geophysical Research, v. 106, p. 13697-13709.

Harris, N. B. W., Inger, S., and Xu, R. H., 1990, Cretaceous plutonism in central Tibet: An example of post-collision magmatism?: Journal of Volcanology and Geothermal Research, v. 44, p. 21-32.

Hoskin, P. W. O., and Schaltegger, U., 2003, The composition of zircon and igneous and metamorphic petrogenesis, in Manchar, J. M., and Hoskin, P. W. O., editors, ZIRCON: Reviews in Mineralogy and Geochemistry, v. 53, p. 27-62.

Koons, P. O., 1995, Modelling the topographic evolution of collisional mountain belts: Annual Review of Earth and Planetary Sciences, v. 23, p. 375-408.

Lee, H. Y., Chung, S. L., Wang, J. R., Wen, D. J., Lo, C. H., Yang, T. Y., Zhang, Y., Xie, Y. W., Lee, T. Y., Wu, G. Y., and Ji, J. Q., 2003, Miocene Jiali faulting and its implications for Tibetan tectonic evolution: Earth and Planetary Science Letters, v. 205, p. 185-194.

Liang, Y. H., Chung, S. L., Liu, D., O'Reilly, S. Y., Chu, M. F., Ji, J. Q., Song, B., and Pearson, N. J., 2004, Detrital zircon study along the Tsangpo River, SE Tibet: Eos Transactions AGU, 85(47), Fall Meeting Abstract T53A-0466.

Lin, I. J., ms, 2007, Geochemical and Sr-Nd isotopic constraints on the petrogenesis of Cretaceous to Paleogene granitoids and volcanic rocks, SE Tibet: National Taiwan University, Masters thesis.

Lin, T. H., Lo, C. H., Chung, S. L., Lee, T. Y., Lee, H. Y. and Hsu, F. J., 2005, New geochronological constraint on the movement of Jiali and Gaoligong shear zones in SE Tibet, and its tectonic implication: Eos Transactions, AGU, 86(52), Fall Meeting Abstract T41A-1281.

Maury, R. C., Pubellier, M., Rangin, C., Wulput, L., Cotton, J., Socquet, A., Bellon, H., Gullaud, J. P., and Htun, H. M., 2004, Quaternary calc-alkaline and alkaline volcanism in a hyper-oblique convergence setting, central Myanmar and western Yunnan: Bulletin de la Société Géologique de France, v. 175 , p. $461-472$.

Mitchell, A. H. G., 1993, Cretaceous-Cenozoic tectonic events in the western Myanmar (Burma)-Assam region: London, Journal of the Geological Society, v. 150, p. 1089-1102.

Mitchell, A. H. G., Htay, M. T., Htun, K. M., Win, M. N., Oo, T., and Hlaing, T., 2007, Rock relationships in the Mogok metamorphic belt, Tatkon to Mandalay, central Myanmar: Journal of Asian Earth Sciences, v. 29, p. 891-910.

Molnar, P., 2003, Nature, nurture, and landscape: Nature, v. 426, p. 612-614.

Najman, Y., 2006, The detrital record of orogenesis: A review of approaches and techniques used in the Himalayan sedimentary basins: Earth-Science Reviews, v. 74, p. 1-72.

Pan, G., Ding, J., Yao, D., and Wang, L., compilers, 2004, The Guide Book of 1: 1,500,000 geologic map of the Qinghai-Xizang (Tibet) plateau and adjacent areas: Chengdu, China, Chengdu Cartographic Publication House, $48 \mathrm{p}$.

Scherer, E., Munker, C., and Mezger, K., 2001, Calibration of the lutetium-hafnium clock: Science, v. 293, p. $683-687$

Schumm, S. A., Dumont, J. E., and Holbrook, J. M., 2000, Active Tectonics and Alluvial Rivers: London, Cambridge University Press, $276 \mathrm{p}$.

Searle, M. P., 2006, Role of the Red River shear zone, Yunnan and Vietnam, in the continental extrusion of SE Asia: London, Journal of the Geological Society, v. 163, p. 1025-1036.

Searle, M. P., Windley, B. F., Coward, M. P., Cooper, D. J. W., Red, A. J., Rex, D. C., Li, T., Xiao, X., Jan, M. Q. Thakur, V. C., and Kumar, S., 1987, The closing of Tethys and the tectonics of the Himalaya: Geological Society of America Bulletin, v. 98, p. 678-701.

Searle, M. P., Noble, S. R., Cottle, J. M., Waters, D. J., Mitchell, A. H. G., Hlaing, T., and Horstwood, M. S. A., 2007, Tectonic evolution of the Mogok metamorphic belt, Burma (Myanmar) constrained by U-Th-Pb dating of metamorphic and magmatic rocks: Tectonics, v. 26, TC3014, doi:10.1029/2006TC002083.

Söderlund, U., Patchett, P. J., Vervoort, J. D., and Isachsen, C. E., 2004. The 176Lu decay constant 
determined by $\mathrm{Lu}-\mathrm{Hf}$ and $\mathrm{U}-\mathrm{Pb}$ isotope systematics of Precambrian mafic intrusions: Earth and Planetary Science Letters, v. 219, p. 311-324.

Stephenson, D., and Marshall, T. R., 1984, The petrology and mineralogy of Mt. Popa volcano and the nature of the late Cenozoic Burma volcanic arc: London, Journal of the Geological Society, v. 141, p. 747-762.

Wang, P. L., Lo, C. H., Lee, T. Y., Chung, S. L., Lan, C. Y., and Nguyen, T. Y., 1998, Thermochronological evidence for the movement of the Ailao Shan-Red River shear zone: A perspective from Vietnam: Geology, v. 26, p. 887-890.

Wen, D. R., ms, 2007, The Gangdese batholith, southern Tibet: Ages, geochemical characteristics and petrogenesis: National Tawian University, Ph. D. thesis.

Wen, D. R., Chung, S. L., Song, B., Ji, J., Liu, D., Qian, Q., Chu, M. F., and Lo, C. H., 2005, The Gangdese batholith: Ages, geochemical characteristics and geodynamic implications: Hangzhou, China, 2nd National Conference on Petrology and Geodynamics Abstracts, p. 399.

Wu, F. Y., Yang, Y. H., Xie, L. W., Yang, J. H., and Xu, P., 2006, Hf isotopic compositions of the standard zircons and baddeleyites used in U-Pb geochronology: Chemical Geology, v. 234, p. 105-126.

Yang, Q. J., Xu, Y. G., Huang, X. L., and Luo, Z. Y., 2006, Geochronology and geochemistry of granites in the Gaoligong tectonic belt, western Yunnan: Tectonic implications: Acta Petrologica Sinica, v. 22, p. $817-$ 834.

Zeitler, P. K., Meltzer, A. S., Koons, P. O., Craw, D., Hallet, B., Chamberlain, C. P., Kidd, W. S. F., Park, S. K. Seeber, L., Bishop, M., and Shroder, J., 2001, Erosion, Himalayan geodynamics, and the geomorphology of metamorphism: GSA Today, v. 11, p. 4-9.

Zhang, P., Molnar, P., and Downs, W. R., 2001, Increased sedimentation rates and grain sizes 2-4 Myr ago due to the influence of climate change on erosion rates: Nature, v. 410, p. 891-987. 